\title{
SO(10) grand unified theories with dynamical Yukawa couplings
}

\author{
Charanjit S. Aulakh ${ }^{* 1,2,3}$ and Charanjit K. Khosa ${ }^{1}$ \\ ${ }^{1}$ Dept. of Physics, Panjab University \\ Chandigarh, 160014, India \\ ${ }^{2}$ Indian Institute of Science Education and Research Mohali, \\ Sector 81, S. A. S. Nagar, Manauli PO 140306, India \\ ${ }^{3}$ Theory Division, CERN, Geneva \\ CH-1213 Geneva 23, Switzerland
}

(Dated: August 1, 2018)

\begin{abstract}
Renormalizable $\mathrm{SO}(10)$ grand unified theories (GUTs), extended by $O\left(N_{g}\right)_{F}$ family gauge symmetry, generate minimal supersymmetric Standard Model flavour structure dynamically via vacuum expectation values of "Yukawon" Higgs multiplets. For concrete illustration and calculability, we work with the fully realistic minimal supersymmetric GUTs based on the $\mathbf{2 1 0} \oplus \overline{\mathbf{1 2 6}} \oplus \mathbf{1 2 6}$ GUT Higgs system - which were already parameter counting minimal relative to other realistic models. $S O(10)$ fermion Higgs channels $\overline{\mathbf{1 2 6}}, \mathbf{1 0}(\mathbf{1 2 0})$ extend to symmetric(antisymmetric) representations of $O\left(N_{g}\right)_{F}$, while $\mathbf{2 1 0 , 1 2 6}$ are symmetric. $N_{g}=3$ dynamical Yukawa generation reduces the matter fermion Yukawas from 15 to 3 (21 to 5) without (with) the $\mathbf{1 2 0}$ Higgs. Yukawon GUTs are thus ultraminimal in parameter counting terms. Consistent symmetry breaking is ensured by a hidden sector Bajc-Melfo(BM) superpotential with a pair of symmetric $O\left(N_{g}\right)$ multiplets $\phi, S$, of which the latter's singlet part $S_{s}$ breaks supersymmetry and the traceless part $\hat{S}$ furnishes flat directions to cancel the $O\left(N_{g}\right)$ D-term contributions of the visible sector. Novel dark matter candidates linked to flavour symmetry arise from both the BM sector and GUT sector minimal supersymmetric Standard Model singlet pseudo-Goldstones. These relics may be viable $\operatorname{light}(<50 \mathrm{GeV})$ cold dark matter as reported by DAMA/LIBRA. In contrast to the new minimal supersymmetric $\mathrm{SO}(10)$ grand unified theory (NMSGUT) even sterile neutrinos can appear in certain branches of the flavour symmetry breaking without the tuning of couplings.
\end{abstract}

\footnotetext{
* aulakh@pu.ac.in; http://physics.puchd.ac.in/aulakh/
} 


\section{INTRODUCTION}

Renormalizable SO(10) GUTs have an array of virtues. 16-plet spinors enclose Standard Model (SM) families with right handed neutrinos required for seesaw neutrino masses. Natural quark lepton unification is achieved with realistic fermion mass relations based on just three allowed fermion mass generating Higgs irreducible representations (irreps) (in the renormalizable case) : $\mathbf{1 0}, \mathbf{1 2 0}, \overline{\mathbf{1 2 6}}$. Matter parity $\left((-)^{3(B-L)}\right)$ is a part of GUT gauge symmetry, and using only vacuum expectation values (VEVs) with even values of Baryon Number $(B)$ - Lepton Number $(L)$ ensures R-parity is conserved to low scales in the supersymmetric case[1]. The lightest supersymmetric particle (LSP) being stable is an ideal weakly interacting massive particle dark matter candidate. SO(10) GUTs based on the $\mathbf{2 1 0} \oplus \overline{\mathbf{1 2 6}} \oplus \mathbf{1 2 6}$ Higgs system [2, 3] are parameter counting minimal[4]. The version called the new minimal supersymmetric $\mathrm{SO}(10)$ grand unified theory (NMSGUT) with the 120plet fits [5-7] all fermion masses and makes distinctive predictions and is thus falsifiable. Notably, we found it requires large soft $A$-terms[6] in 2008 : well before Higgs discovery promoted general acceptance of this possibility. It also predicts a normal s-hierarchy, Bino LSP and can yield a light smuon as the next to lightest supersymmetric particle(NLSP), thus promoting a Bino as a good dark matter candidate and making a significant supersymmetric contribution to the muon magnetic moment anomaly. Recently, we showed that careful attention to the quantum communication between the UV completion and its effective theory through the light Higgs portal yields natural and generic suppression of fast proton decay in supersymmetric(SUSY) GUTs[7].

The appealing speculation that the observed dimensionless fermion Yukawa couplings actually arise via VEVs of 'spurion' fields has a long history[8]. In Ref.[9], these fields appropriately called "Yukawa-on"s- carry representations of $U\left(N_{g}\right)^{6}$ family symmetry (in a theory with six SM flavours $\left(Q, L, u_{L}^{c}, d_{L}^{c}, e_{L}^{c}, \nu_{L}^{c}\right)$ and $N_{g}$ generations), but not of the SM gauge group. Given that the main hint for flavour unification is the convergence of third generation Yukawas at GUT scales, Yukawon models with flavour symmetry broken at the GUT scale are well motivated. Our work [2, 4 -7, 10] on realistic renormalizable supersymmetric SO(10) GUTs - which encodes the fermion hierarchy in the SO(10) matter fermion Yukawa matrices and pays careful attention to how the minimal supersymmetric SM (MSSM) Higgs emerges from the multiple MSSM type doublets present in the UV 
theory-also naturally and minimally identifies the associated Higgs multiplets as candidate Yukawon multiplets. It thus yields a novel mechanism whereby the fermion hierarchy could emerge from a flavour symmetric and renormalizable GUT . Thus, in our work "Yukawons" also carry representations of the gauge $(S M / G U T)$ dynamics. In previous work, typically the dimension 1 Yukawa-on $\mathcal{Y}$ in the Higgs vertex made it non-renormalizable $\left(\mathcal{L}=f^{c} \mathcal{Y} f H / \Lambda_{\mathcal{Y}}+\right.$ ...), where the unknown high scale $\Lambda_{\mathcal{V}}$ controls Yukawa-on dynamics. In this paper, we work out how minimal SO(10) GUTs [2 4, 6] provide a gauged $O\left(N_{g}\right)$ family symmetry route to "Yukawonifcation" : with the GUT and family symmetry breaking at the same scale: obviating the need for non-renormalizable interactions and any extraneous scale $\Lambda_{\mathcal{Y}}$. As we shall see, the consistency conditions for the maintainability of the Higgs portal to the UV completion of the MSSM play a central role in determining just how the peculiarly lopsided and "senseless" fermion hierarchy is produced from the flavour symmetric and grand unified UV completion. In our [2, 4 7] minimal SUSY GUTs, we eschew invocation of discrete symmetries and insist only upon following the logic of $\mathrm{SO}(10)$ gauge symmetry. This insistence, combined with careful attention to the implications of the emergence of a light MSSM Higgs pair from the $2 N_{g}\left(N_{g}+1\right)$ pairs in the $O\left(N_{g}\right)$ extended minimal supersymmetric GUT(MSGUT), leads to an effectively unique extension of the $\mathrm{SO}(10)$ gauge group by a $O\left(N_{g}\right)$ family gauge symmetry for the $N_{g}$ generation case and the dynamical emergence of fermion hierarchy and mixing.

The plan of this paper is as follows: In Section II we explain the basic mechanism of generation of Flavor hierarchy by enforcement of the masslessness of MSSM Higgs. In Section III we discuss the spontaneous symmetry breaking of the GUT and flavor symmetries. In Section IV we show how to calculate the flavor hierarchies associated with two generation (toy model) and three generation (realistic case) example solutions.In Section V we discuss our results and the research program they define. In Appendix A we give the explicit values of the VEVs for our example solutions. In Appendix B we give the numerical values of the Yukawa hierarchies for the three cases studied in Section IV along with the complete superheavy spectra for each case. 


\section{MSGUTS AND THE YUMGUT PROPOSAL}

The minimal SUSY SO(10) model[2, 3] consists of an SM singlet containing Higgs irreps, $(\mathbf{2 1 0}(\Phi) \oplus \overline{\mathbf{1 2 6}}(\bar{\Sigma}) \oplus \mathbf{1 2 6}(\boldsymbol{\Sigma}))$, responsible for GUT symmetry breaking and right handed

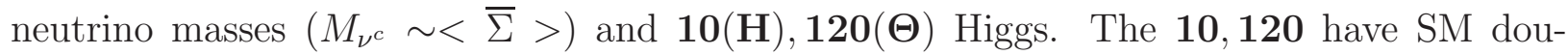
blets but no SM singlets and thus do not participate in GUT scale spontaneous symmetry breaking (SSB). The $\overline{\mathbf{1 2 6}}(\overline{\mathbf{\Sigma}})$ also contributes to both neutrino and charged fermion masses. Gauging just an $O\left(N_{g}\right)$ subgroup of the $U\left(N_{g}\right)$ symmetry of the fermion kinetic terms is workable because the use of complex representations of a unitary family group introduces anomalies and requires doubling of the Higgs structure to cancel anomalies and to permit holomorphic invariants to be formed for the superpotential. Worse, unitary symmetry enforces vanishing of half the emergent matter Yukawa couplings. $O\left(N_{g}\right)$ family symmetry suffers from none of these defects and gauging it ensures that no Goldstone bosons arise when it is spontaneously broken. $O(3)$ family symmetry with tracefull six-dimensional irrep has also been considered for non-renormalizable, non-GUT Yukawa-on [9] models. However, our model is renormalizable and GUT based and thus radically different in its construction and implications.

The GUT superpotential has exactly the same form as the MSGUT (see Refs. 6, 10 12] for comprehensive details) :

$$
\begin{aligned}
W_{G U T} & =\operatorname{Tr}\left(m \Phi^{2}+\lambda \Phi^{3}+M \bar{\Sigma} \cdot \Sigma+\eta \Phi \cdot \bar{\Sigma} \cdot \Sigma\right) \\
& \left.+\Phi \cdot H \cdot(\gamma \Sigma+\bar{\gamma} \cdot \bar{\Sigma})+M_{H} H \cdot H\right) \\
W_{F} & =\Psi_{A} \cdot((h H)+(f \Sigma)+(g \Theta))_{A B} \Psi_{B}
\end{aligned}
$$

We have shown how the 120-plet is included in $W_{F}$ but will here study only MSGUTs (i.e with $10, \overline{\mathbf{1 2 6}}$ ). Inclusion of the $\mathbf{1 2 0}$-plet does not affect GUT SSB. The only innovation in Higgs structure is that all the MSGUT Higgs fields now carry symmetric representation of the $O\left(N_{g}\right)$ family symmetry : $\{\Phi, \bar{\Sigma}, \Sigma, H\}_{A B} ; A, B=1,2 . . N_{g}$ (under which the matter 16-plets $\psi_{A}$ are vector $N_{g}$-plets). Couplings $h, f, g$ are single complex numbers while the Yukawons carry symmetric $(\mathbf{H}, \overline{\boldsymbol{\Sigma}})$ and antisymmetric $\left(\boldsymbol{\Theta}_{\mathbf{A B}}\right)$ representations of $O(3)$ : as required by the transposition property of relevant $\mathrm{SO}(10)$ invariants. For $N_{g}=3$, real fermion mass parameters come down from $15\left(R e\left[h_{A A}\right], f_{A B}\right)$ to just $3(R e[h], f)$ without the 120-plet (6 additional to just 2 with the 120-plet). Thus this type of renormalizable flavour 
unified GUTs can legitimately be called a Yukawon ultraminimal GUTs(YUMGUTs).

An essential component of our proposal is that the single pair of light Higgs doublets $\bar{H}, H$ in the effective MSSM arise as combinations of the manifold pairs of MSSM doublets contained in the full set of fields. The consistency(also known as "fine -tuning") conditions for obtaining a light pair are here seen positively as the "open sesame" to peek through the Higgs portal into the UV completion. This is possible precisely because the effective light pair is so deeply entangled in the innards of the UV theory in spite of being a bonafide member of the low-energy effective theory i.e the MSSM. After imposing Det $\mathcal{H}=0$ on the doublet mass matrix $\mathcal{H}$, one pair, $\overline{\mathbf{H}}[1,2,-1] \oplus \mathbf{H}[1,2,1]$, remains light. Its Yukawa couplings are determined by the "Higgs-fractions" specified by the left and right null eigenvectors of $\mathcal{H}\left[4,[6,10]\right.$. $\mathcal{H}$ depends on the family symmetry breaking $\operatorname{SM}$ singlet $\operatorname{VEVs}(p, a, \omega)_{A B} \in<$ $\Phi_{A B}>,(\sigma, \bar{\sigma})_{A B} \in<\Sigma, \bar{\Sigma}>_{A B}$. Here, for simplicity, we ignore the 120-plet, although it is known that this multiplet is required for fully realistic fermion masses when there is no family symmetry [5, 6]. Then the four- dimensional $\mathcal{H}$ of the MSGUT becomes $2 N_{g}\left(N_{g}+1\right)$ dimensional. If the $\mathbf{1 2 0}$-plet were included (NMSGUT), $\mathcal{H}$ would have dimension $N_{g}\left(3 N_{g}+\right.$ 1).

$\mathcal{H}=\left(\begin{array}{cccc}-M_{H} & \bar{\gamma} \sqrt{3} \Omega(\omega-a) & -\gamma \sqrt{3} \Omega(\omega+a) & -\bar{\gamma} \Omega(\bar{\sigma}) \\ \gamma \sqrt{3} \Omega(\omega-a) & -(2 M+4 \eta \Omega(a-\omega)) & \varnothing_{d} & -2 \eta \sqrt{3} \Omega(\bar{\sigma}) \\ -\bar{\gamma} \sqrt{3} \Omega(\omega+a) & \varnothing_{d} & -(2 M+4 \eta \Omega(\omega+a)) & \varnothing_{d} \\ -\gamma \Omega(\sigma) & -2 \eta \sqrt{3} \Omega(\sigma) & \varnothing_{d} & -2 m+6 \lambda \Omega(\omega-a)\end{array}\right)$

$\varnothing_{d}$ is the $\mathrm{d}$ dimensional null square matrix. The rows are labelled by the $N_{g}\left(N_{g}+1\right) / 2$-tuples (ordered and normalized, for a symmetric $\phi_{A B}, A, B=1 . . N_{g}$, as $\left.\left\{\phi_{11}, \phi_{22}, \ldots \phi_{N_{g} N_{g}}, \sqrt{2} \phi_{12}, \sqrt{2} \phi_{13}, \ldots ., \sqrt{2} \phi_{N_{g}-1, N_{g}}\right\}\right)$

containing MSSM type $\bar{H}[1,2,-1]$ doublets from 10, 126, $\overline{\mathbf{1 2 6}}, \mathbf{2 1 0}$. The columns are labelled by $H[1,2,1]$ doublets in the order $\mathbf{1 0}, \overline{\mathbf{1 2 6}}, \mathbf{1 2 6}, \mathbf{2 1 0}$. The matrix function $\Omega(\phi)$ has the form determined by the symmetric invariant $\operatorname{Tr}[<V>. \bar{H} \cdot H]$. For $N_{g}=2$, it is

$$
\Omega_{2}[V]=\left(\begin{array}{ccc}
V_{11} & 0 & V_{12} / \sqrt{2} \\
0 & V_{22} & V_{12} / \sqrt{2} \\
V_{12} / \sqrt{2} & V_{12} / \sqrt{2} & \left(V_{11}+V_{22}\right) / 2
\end{array}\right)
$$


with labels $\left\{\bar{H}_{11}, \bar{H}_{22}, \sqrt{2 H}_{12}\right\} \oplus\left\{H_{11}, H_{22}, \sqrt{2} H_{12}\right\}$. Imposing Det $\mathcal{H}=0$, fermion Yukawa couplings of MSSM are extracted by generalizing the MSGUT procedure [4, 6, 10, 11]. For $N_{g}=2$, we get Yukawa couplings

$$
\begin{aligned}
& Y_{u}=\left(\begin{array}{cc}
\hat{h} \hat{V}_{1}+\hat{f} \hat{V}_{4} & \left(\hat{h} \hat{V}_{3}+\hat{f} \hat{V}_{6}\right) / \sqrt{2} \\
\left(\hat{h} \hat{V}_{3}+\hat{f} \hat{V}_{6}\right) / \sqrt{2} & \hat{h} \hat{V}_{2}+\hat{f} \hat{V}_{5}
\end{array}\right) \\
& Y_{d}=\left(\begin{array}{cc}
\hat{h} \hat{W}_{1}+\hat{f} \hat{W}_{7} & \left(\hat{h} \hat{W}_{3}+\hat{f} \hat{W}_{9}\right) / \sqrt{2} \\
\left(\hat{h} \hat{W}_{3}+\hat{f} \hat{W}_{9}\right) / \sqrt{2} & \hat{h} \hat{W}_{2}+\hat{f} \hat{W}_{8}
\end{array}\right) \\
& \hat{h}=2 \sqrt{2} h \quad ; \quad \hat{f}=-4 i \sqrt{\frac{2}{3}} f
\end{aligned}
$$

The Yukawas $Y_{\nu}, Y_{l}$ for the neutrinos and charged leptons are obtained from $Y_{u}, Y_{d}$ by the replacement $\hat{f} \rightarrow-3 \hat{f} . \hat{V}, \hat{W}$ are the normalized right and left null eigenvectors of the mass matrix $\mathcal{H}$ and contain[10, 11] the "Higgs fractions". It is obvious that $|h|>>|f|$ implies $Y_{u}=Y_{\nu}, Y_{d}=Y_{l}$. Right-handed neutrino masses are proportional to $\left.f<\bar{\Sigma}\right\rangle$, so this condition will elevate type I (and depress type II) seesaw light neutrino masses. On the other hand, the type II seesaw mechanism is more complicated now since the $O(1,3,-2)$ $S U(2)_{L}$ triplet sub-multiplets of $\overline{\mathbf{1 2 6}}$ ( the VEVs of which generate type II seesaw masses) are now symmetric multiplets of the flavour group. Although the same kind of conflicting demands on the magnitude of the coupling $f$ that put paid to the MSGUT's claim to fit neutrino masses[5]may well operate here it must actually be verified in detail. Our results here even suggest that when light sterile neutrinos are present the achieved neutrino masses may be much larger: to our knowledge this possibility was not considered earlier in MSGUTs. Since our objective in this paper is not the actual fitting of the observed masses but only the introduction of flavour-gauged MSGUTs and the way in which they generate MSSM couplings, we postpone the computation of type II seesaw masses to a sequel. We will however give numerical illustrations of the difficulty with type I.

\section{SPONTANEOUS SYMMETRY BREAKING IN YUMGUTS}

To demonstrate the feasibility of our proposal for dynamical fermion flavour mixing and mass hierarchy from a family symmetric Lagrangian, we must show the GUT scale spontaneous symmetry breaking leads to Yukawas like those of the observed fermion hierarchy. Of course such a program will be complete only after finding values of the -flavour bland- 
GUT parameter set compatible with all other requirements such as gauge coupling unification, proton stability and constraints on exotic processes such as quark and lepton flavour violation, cosmology etc. Without family symmetry this was achieved, apart from a failure in matching neutrino masses [5, 10], in the MSGUT and, after prolonged investigations during 2006-2013, fully in the NMSGUT [6, 7]. We are now attempting to build upon those successes by trying to generate also flavour structure on the basis of the GUT SSB which is flavour blind. When carrying this out at the fully realistic level we will need to search the -flavour blind and thus much reduced- parameter set of the YUMGUT for sets of parameters that yield fully realistic Yukawas. The Yukawonification searches will have much larger mass matrices than the (N)MSGUT case but are also simpler due to the reduced parameter space. However, before embarking upon this mammoth and exacting project, we wish to show that even for generic values of parameters, the flavour structure that emerges can have the main features (hierarchy, small quark mixing, large lepton mixing) actually observed. Thus here we only indicate the operative structure, and show feasibility by finding GUT scale SSB solutions for generic values of flavour bland parameters that yield a fermion hierarchy qualitatively similar to that required in actuality. The search for realistic parameter sets is a long-term objective that is motivated and justified by the in-principle feasibility we demonstrate here for both $N_{g}=2$ and $N_{g}=3$.

Giving VEVs [4, 10, 11] to the SM singlets $p, a, \omega$ of the $\left(\mathbf{2 1 0}, d_{s}\left[N_{g}\right]\right)$-plet and the $\sigma \in$ (126, $\left.d_{s}\left[N_{g}\right]\right), \bar{\sigma} \in\left(\overline{\mathbf{1 2 6}}, d_{s}\left[N_{g}\right]\right)$, where $d_{s}\left[N_{g}\right]=N_{g}\left(N_{g}+1\right) / 2$, the superpotential relevant to GUT SSB has just the MSGUT form, with sums over flavour group indices:

$$
\begin{aligned}
W= & \operatorname{Tr}\left[m\left(p^{2}+3 a^{2}+6 \omega^{2}\right)+2 \lambda\left(a^{3}+3 p \omega^{2}+6 a \omega^{2}\right)\right] \\
& +\operatorname{Tr}\left[M \bar{\sigma} \sigma+\eta(p+3 a-6 \omega) \frac{(\bar{\sigma} \sigma+\sigma \bar{\sigma})}{2}\right]
\end{aligned}
$$

In the MSGUT case, the entire SSB problem reduces[4] (i.e for $N_{g}=1$ in present notation) to a cubic equation for a single complex variable $x=-\omega$ (with just one free parameter $\xi=M \lambda / m \eta)$. YUMGUTs proposed here generalize that solution to the family symmetric case. Visible sector F-terms give :

$$
\begin{gathered}
2 m(p-a)-2 \lambda a^{2}+2 \lambda \omega^{2}=0 \\
2 m(p+\omega)+\lambda(p+2 a+3 \omega) \omega+\lambda \omega(p+2 a+3 \omega)=0 \\
M \sigma+\eta(\chi \sigma+\sigma \chi) / 2=0
\end{gathered}
$$




$$
\begin{gathered}
M \bar{\sigma}+\eta(\chi \bar{\sigma}+\bar{\sigma} \chi) / 2=0 \\
\bar{\sigma} \sigma+\sigma \bar{\sigma}=-\frac{4}{\eta}\left(m p+3 \lambda \omega^{2}\right) \equiv F
\end{gathered}
$$

where $\chi \equiv(p+3 a-6 \omega)$. Homogenous equations (89) can be put in the form $\boldsymbol{\Xi} \cdot \hat{\boldsymbol{\Sigma}}=\boldsymbol{\Xi} \cdot \hat{\bar{\Sigma}}=\mathbf{0}$, where $\hat{\boldsymbol{\Sigma}}, \hat{\bar{\Sigma}}$ are $N_{g}\left(N_{g}+1\right) / 2$ dimensional vectors : e.g. for $N_{g}=2, \hat{\boldsymbol{\Sigma}}=$ $\left\{\sigma_{11}, \sigma_{22}, \sigma_{12}\right\}$, the matrix $\boldsymbol{\Xi}$ involves the combinations $\chi_{A}=\chi_{A A}+\frac{M}{\eta}$ :

$$
\boldsymbol{\Xi}=\left(\begin{array}{ccc}
\chi_{1} & 0 & \chi_{12} \\
0 & \chi_{2} & \chi_{12} \\
\chi_{12} & \chi_{12} & \chi_{1}+\chi_{2}
\end{array}\right)
$$

Nontrivial solutions of Eq. (8) and (9) for $\sigma, \bar{\sigma}$ exist only if $\operatorname{Det}[\boldsymbol{\Xi}]=0$. Once Eqs. (6)-(10) are (numerically) solved, there remain only the D-term conditions $D_{B-L}=0$ (the only nontrivial condition from the vanishing of $\mathrm{SO}(10) \mathrm{D}$ terms) and $D^{A}=0$ from $O\left(N_{g}\right)$. In the MSGUT $D_{B-L}=0$ requires $|\sigma|=|\bar{\sigma}|$ and since $\operatorname{Arg}[\sigma]-\operatorname{Arg}[\bar{\sigma}]$ can be gauged away by $U(1)_{B-L}$, the D-terms supplement F-terms to a nicety. We shall retain this convenience by only considering solutions with $\sigma_{A B}=\bar{\sigma}_{A B}$.

At this point, an obstacle arises. $O\left(N_{g}\right)$ D-terms vanish for vanishing visible sector $\mathrm{F}$ terms only for trivial(flavour diagonal) solutions, since different flavour charge sectors have no reason to cancel. So additional fields with VEVs free to cancel the contribution $\bar{D}_{X}^{A}$ of the GUT sector to $O\left(N_{g}\right)$ D terms are needed. The extra F terms must be sequestered from the GUT sector to preserve the MSGUT SSB. We propose[13] Bajc-Melfo two field superpotentials [14, 15] ( of structure $W=S\left(\mu_{B} \phi+\lambda_{B} \phi^{2}\right)$ ). Their potentials have local minima breaking supersymmetry $\left(<F_{S}>\neq 0\right)$, which leave the $\mathrm{VEV}<S>$ undetermined ( $\phi$ gets a VEV). Radiative corrections that determine $\langle S>$ then provide an alternative realization[15] of the Witten[16] model for high-scale symmetry breaking triggered by a low scale symmetry breaking. Determination of the flat direction VEV by $\mathrm{N}=1$ supergravity corrections instead of radiative effects was also shown to be effective long ago[17, 18].

In Ref.[13] we show that coupling Bajc-Melfo fields to supergravity resolves the flat direction and fixes the $\mathrm{VEV}<S_{s}>\sim M_{p}$. If $S, \phi$ are also traceful symmetric multiplets $(S, \phi)_{A B}$ of $O\left(N_{g}\right)$, then the traceless symmetric multiplet $\left(\hat{S}_{A B}\right)$ is also undetermined at tree level and free to cancel $\bar{D}_{X}^{A}$. One gets a gravity-mediated scenario in which the hidden sector breaking involves breaking of family symmetry and supersymmetry. The additional fields include moduli like fields $\left(\hat{S}_{A B}\right)$, which can be light enough to serve as light dark 
matter with mass less than $50 \mathrm{GeV}$. Such masses are favoured [19] by experiments such as DAMA/LIBRA but were unobtainable in MSGUTs earlier. They are signals of the hidden (sector) connection between supersymmetry and family symmetry breaking. Polonyi and moduli problems [20] may be evaded because of the rich Yukawa and gauge dynamics in the hidden sector. We assume results of Ref. [13], which were designed to ensure that the $O\left(N_{g}\right)$ terms can be cancelled and supersymmetry broken.

\section{SOLUTIONS WITH FLAVOUR GENERATION}

For $N_{g}>1$, one must consider the cases that arise separately. For $N_{g}=2$

$$
\begin{aligned}
& \operatorname{Det}[\boldsymbol{\Xi}]=\left(\chi_{1}+\chi_{2}\right)\left(\chi_{12}^{2}-\chi_{1} \chi_{2}\right)=0 \\
& \Rightarrow \quad \chi_{1}=-\chi_{2} \quad \text { OR } \quad \chi_{12}= \pm \sqrt{\chi_{1} \chi_{2}}
\end{aligned}
$$

If $2 \times 2$ minors of $\boldsymbol{\Xi}$ also vanish then additionally $\left(\chi_{1}+\chi_{2}\right) \chi_{1}=\left(\chi_{1}+\chi_{2}\right) \chi_{2}=\chi_{12}^{2}$ and $\chi_{1} \chi_{2}=0$. Then $\chi_{1}=\chi_{2}=\chi_{12}=0$ so that $\boldsymbol{\Xi} \equiv 0$. Thus, $\operatorname{Rank}[\boldsymbol{\Xi}]<2$ implies $\operatorname{Rank}[\boldsymbol{\Xi}]=0$ so that none of the six $\sigma, \bar{\sigma}$ are determined. In the $\operatorname{Rank}[\boldsymbol{\Xi}]=0$ case, one ultimately finds that the GUT spectra in sectors that include $\left(S O(10) / G_{123}\right)$ coset gauginos always include large colour and electroweak non-singlet pseudo-Goldstone multiplets that would ruin unification. Therefore, we focus on the non degenerate cases in which $\operatorname{Det}[\boldsymbol{\Xi}]=0$ but $\operatorname{Rank}[\boldsymbol{\Xi}]=2$ for $N_{g}=2$ and $\operatorname{Rank}[\boldsymbol{\Xi}] \leq 5$ for $N_{g}=3$. To show that our scenario is at least prima facie consistent, in Appendix A we give solutions of Eqs.(6)-(10) with zero $D_{B-L}$ for $N_{g}=2, N_{g}=3$ and the corresponding value of $\bar{D}_{X}$ for a set of YUMGUT flavour bland parameters (similar to those shown to be applicable in realistic fits in the NMSGUT [7]). We also give the complete GUT mass spectrum (in units of $m / \lambda$ ) so as to show that although there are cases with acceptable fermion hierarchies where there are light sterile neutrinos and light MSSM singlets that can be interesting dark matter candidates, these are not plagued by MSSM non singlet pseudo-Goldstone chiral supermultiplets which would ruin the gauge coupling unification so central to the whole NMSGUT project. In this section, we explain some of the choices we made while navigating the algebraic complexity of finding solutions of the coupled highly non-linear equations (6)-(10). First, as always in MSGUTs [4], it is convenient to work in units of $m / \lambda:\{P, W, A, \tilde{\sigma}, \tilde{\bar{\sigma}}, \tilde{\chi}\}=\frac{\lambda}{m}\{p, \omega, a, \sigma, \bar{\sigma}, \chi\}$, $\tilde{\chi}_{A}=\tilde{\chi}_{A A}+\xi$ which removes most of the parameter clutter in favour of just two parameter 
ratios $(\xi=\lambda M / \eta m$ and $\lambda / \eta)$.

$$
\begin{gathered}
2\left(\frac{m}{\lambda}\right)^{2}\left(P-A-A^{2}+W^{2}\right)=0 \\
\left(\frac{m}{\lambda}\right)^{2}(P+W+(P+2 A+3 W) W+W(P+2 A+3 W))=0 \\
\left(\frac{m}{\lambda}\right)^{2}(\xi \tilde{\sigma}+(\tilde{\chi} \tilde{\sigma}+\tilde{\sigma} \tilde{\chi}) / 2)=0 \\
\left(\frac{m}{\lambda}\right)^{2}(\xi \tilde{\bar{\sigma}}+(\tilde{\chi} \tilde{\bar{\sigma}}+\tilde{\bar{\sigma}} \tilde{\chi}) / 2)=0 \\
\tilde{\bar{\sigma}} \tilde{\sigma}+\tilde{\sigma} \tilde{\bar{\sigma}}=-\frac{4 \lambda}{\eta}\left(P+3 W^{2}\right)=\frac{\lambda^{2} F}{m^{2}}=\tilde{F}
\end{gathered}
$$

From now on, we will use these dimensionless equations for SSB analysis.

\section{A. $N_{g}=2, \operatorname{Rank}[\boldsymbol{\Xi}]=2$}

As explained earlier, we will always take $\tilde{\sigma}=\tilde{\bar{\sigma}}$. $\operatorname{Det}[\boldsymbol{\Xi}]$ (same form as earlier but now in terms of dimensionless VEVs) has two factors $\left(\tilde{\chi}_{1}+\tilde{\chi}_{2}\right)$ and $\left(\tilde{\chi}_{12}^{2}-\tilde{\chi}_{1} \tilde{\chi}_{2}\right)$, and one out of these should vanish. From $\boldsymbol{\Xi} \cdot \hat{\boldsymbol{\Sigma}}=\mathbf{0}$, we can calculate $\tilde{\sigma}_{11}$ and $\tilde{\sigma}_{22}$ in terms of $\tilde{\sigma}_{12}$.

$$
\begin{gathered}
\tilde{\sigma}_{11}=-\frac{\tilde{\chi}_{12}}{\tilde{\chi}_{1}} \tilde{\sigma}_{12} ; \quad \tilde{\sigma}_{22}=-\frac{\tilde{\chi}_{12}}{\tilde{\chi}_{2}} \tilde{\sigma}_{12} \\
\operatorname{Det}[\tilde{\sigma}]=\frac{\left(\tilde{\chi}_{12}^{2}-\tilde{\chi}_{1} \tilde{\chi}_{2}\right)}{\tilde{\chi}_{1} \tilde{\chi}_{2}} \tilde{\sigma}_{12}^{2}
\end{gathered}
$$

Notice the existence of one common factor in $\operatorname{Det}[\boldsymbol{\Xi}]$ and $\operatorname{Det}[\tilde{\sigma}]$. For a type I seesaw to operate for all neutrino masses, we need an invertible $\overline{\mathbf{1 2 6}} \mathrm{VEV}$ since the Majorana mass matrix of the right-handed neutrinos is proportional to $\langle\bar{\Sigma}\rangle$. Thus, we consider only the branch $\left(\tilde{\chi}_{1}+\tilde{\chi}_{2}\right)=0$ for vanishing $\operatorname{Det}[\boldsymbol{\Xi}]$. Then, $\tilde{\sigma}^{2}$ is diagonal. Equation (17) then implies

$$
\begin{aligned}
\tilde{\sigma}_{11}^{2}= & =\frac{\tilde{F}_{11} \tilde{\chi}_{12}^{2}}{2\left(\tilde{\chi}_{12}^{2}+\tilde{\chi}_{1}^{2}\right)} \\
\tilde{F}_{11} & =\tilde{F}_{22} \quad ; \quad \tilde{F}_{12}=0
\end{aligned}
$$

We begin by solving the three equations in Eq.(14), which is linear in $P$ and parameter free, for the three components of $P$. Using these values $\tilde{\chi}_{1}=-\tilde{\chi}_{2}$ i.e

$$
\left(P_{11}+3 A_{11}+W_{11}\right)-\left(P_{22}+3 A_{22}+W_{22}\right)+2 \xi=0
$$


is solved for $A_{11}$ and then $\tilde{F}_{12}=0$ for $A_{12}$ and finally $\tilde{F}_{11}-\tilde{F}_{22}=0$ for $A_{22}$. Then, we solve the remaining equations(13), which are now expressed in terms of $W$ and $\xi$. We solve numerically for $W$ for convenient $\xi$ using a minimization method.

With $P, A, W, \tilde{\sigma}$ in hand (see Appendix A for numerical values of these for sample sets of YUMGUT parameters), we find the $N_{g}\left(N_{g}+1\right) / 2$ values of $M_{H}$ for which Det $\mathcal{H}=0$. For each of these possible values, we can diagonalize MSSM matter Yukawas (4) and the neutrino mass matrices to determine the fermion hierarchy. Of course, the light neutrino masses will depend not only on the electroweak VEV and $\tan \beta$ in the effective MSSM but also on the masses of the right-handed neutrinos that depend on the Grand Unification scale [4, 10, 11]. Here, we wish only to illustrate that fermion hierarchies qualitatively similar to those observed in reality can arise for quite generic values of the YUMGUT parameters.

The fermion hierarchies we obtain in the $N_{g}=2$ case for $f \sim 10^{-1}$ and for $f \sim 10^{-4}$ are exhibited in Table \and \In Appendix B. We see that while $f \sim h$ generates acceptable quark and lepton mixing the neutrino masses and mass splitting squared tend to be much too small as is clear from Table I. On the other hand-as seen in Table II when we lower $f$ we can raise the type I seesaw neutrino masses to an acceptable level but we then lose acceptable Yukawa couplings obtained for $f \sim h$. To close the argument for $f \sim h$, one needs to look also at the operation of type II seesaw. However, note that in the MSGUT type II was always sub dominant to type I because the mass of $O_{-}[1,3,-2]$ is generically of GUT scale whereas the largest right-handed neutrino masses allowed by type I seesaw are of order $10^{13-14} \mathrm{GeV}$. Even though we now have a triplet of $O_{-}[1,3,-2]$ fields, except at special points, we may expect the same large masses to suppress the type II seesaw masses far below type I masses. Still, detailed study may reveal interesting special cases. Generically, however, the above arguments lead us to expect that introduction of the 120-plet and a special scenario involving the lowering effect of threshold corrections on down type quark masses of the first two generations [5, 6] is necessary for phenomenological viability.

Thus, the main point of this numerical exercise with a toy model is that without any attempt at optimizing these parameters, we find "small" quark and "large" lepton mixing angles and a hierarchy between generations by factors of 10. Such values in the pivotal[21] 23 sector inspire confidence that an optimizing search for flavour-bland GUT parameters may do much better.

A further issue that we must consider is the possibility that the mass spectrum of the 26 
different MSSM multiplet types that occur in YUMGUTs based upon the MSGUT contains unacceptable pseudo-Goldstone multiplets which would ruin coupling unification. In Table III, we exhibit the spectrum in units of the superheavy mass $m / \lambda \sim 10^{16} \mathrm{GeV}$. It is seen that there is indeed a Pseudo-Goldstone of multiplet type G(see Refs. [10, 11] for notation) with MSSM quantum numbers $[1,1,0]$. However, since this is a gauge singlet, it does not affect the evolution of the MSSM gauge couplings or their unification. Such particles are novel candidates for dark matter.

\section{B. $N_{g}=3$ SSB}

The symmetry breaking equations for the realistic case $N_{g}=3$ are considerably more complex to solve but the patterns they yield are again of the right type and moreover they introduce a panoply of possibilities including cases with light sterile neutrinos. To see how these arise, note that once again $\tilde{\sigma}$ and $\tilde{\bar{\sigma}}$ equations can be written as

$$
\Xi \cdot \hat{\Sigma}=\Xi \cdot \hat{\bar{\Sigma}}=0
$$

Now

$$
\boldsymbol{\Xi}=\left(\begin{array}{cccccc}
\tilde{\chi}_{1} & 0 & 0 & \tilde{\chi}_{12} & \tilde{\chi}_{13} & 0 \\
0 & \tilde{\chi}_{2} & 0 & \tilde{\chi}_{12} & 0 & \tilde{\chi}_{23} \\
0 & 0 & \tilde{\chi}_{3} & 0 & \tilde{\chi}_{13} & \tilde{\chi}_{23} \\
\tilde{\chi}_{12} & \tilde{\chi}_{12} & 0 & \tilde{\chi}_{1}+\tilde{\chi}_{2} & \tilde{\chi}_{23} & \tilde{\chi}_{13} \\
\tilde{\chi}_{13} & 0 & \tilde{\chi}_{13} & \tilde{\chi}_{23} & \tilde{\chi}_{1}+\tilde{\chi}_{3} & \tilde{\chi}_{12} \\
0 & \tilde{\chi}_{23} & \tilde{\chi}_{23} & \tilde{\chi}_{13} & \tilde{\chi}_{12} & \tilde{\chi}_{2}+\tilde{\chi}_{3}
\end{array}\right)
$$

and $\hat{\Sigma}=\left\{\tilde{\sigma}_{11}, \tilde{\sigma}_{22}, \tilde{\sigma}_{33}, \tilde{\sigma}_{12}, \tilde{\sigma}_{13}, \tilde{\sigma}_{23}\right\}$

$$
\begin{aligned}
\operatorname{Det}[\boldsymbol{\Xi}]= & \left(\tilde{\chi}_{1} \tilde{\chi}_{2} \tilde{\chi}_{3}-\tilde{\chi}_{1} \tilde{\chi}_{23}^{2}-\tilde{\chi}_{12}^{2} \tilde{\chi}_{3}+2 \tilde{\chi}_{12} \tilde{\chi}_{13} \tilde{\chi}_{23}-\tilde{\chi}_{13}^{2} \tilde{\chi}_{2}\right) \\
& \left(\tilde{\chi}_{1}^{2} \tilde{\chi}_{2}+\tilde{\chi}_{1}^{2} \tilde{\chi}_{3}-\tilde{\chi}_{1} \tilde{\chi}_{12}^{2}-\tilde{\chi}_{1} \tilde{\chi}_{13}^{2}+\tilde{\chi}_{1} \tilde{\chi}_{2}^{2}+2 \tilde{\chi}_{1} \tilde{\chi}_{2} \tilde{\chi}_{3}+\tilde{\chi}_{1} \tilde{\chi}_{3}^{2}\right. \\
& \left.-\tilde{\chi}_{12}^{2} \tilde{\chi}_{2}-2 \tilde{\chi}_{12} \tilde{\chi}_{13} \tilde{\chi}_{23}-\tilde{\chi}_{13}^{2} \tilde{\chi}_{3}+\tilde{\chi}_{2}^{2} \tilde{\chi}_{3}-\tilde{\chi}_{2} \tilde{\chi}_{23}^{2}+\tilde{\chi}_{2} \tilde{\chi}_{3}^{2}-\tilde{\chi}_{23}^{2} \tilde{\chi}_{3}\right)
\end{aligned}
$$

For a nontrivial solution to exist, $\operatorname{Det}[\boldsymbol{\Xi}]=0$ is necessary. We first discuss the non degenerate case $\operatorname{Rank}[\boldsymbol{\Xi}]=5$. 


\section{1. $\operatorname{Rank}[\boldsymbol{\Xi}]=5$}

By Kramer's rule, we can determine five of the $\tilde{\sigma}$ variables in terms of one undetermined variable (say $\left.\tilde{\sigma}_{23}\right)$. From Eq. (22)

$$
\left(\begin{array}{cc}
\Xi_{5} & v \\
v^{T} & \tilde{\chi}_{2}+\tilde{\chi}_{3}
\end{array}\right)\left(\begin{array}{c}
\hat{\sigma} \\
\tilde{\sigma}_{23}
\end{array}\right)=0 \quad \Rightarrow \quad \hat{\sigma}=-\left(\Xi_{5}^{-1} v\right) \tilde{\sigma}_{23}
$$

Here, $\Xi_{5}$ and $v$ are the upper left $5 \times 5$ block and sixth column (less the 66 element) of $\boldsymbol{\Xi}$ respectively. $\hat{\sigma}=\left(\tilde{\sigma}_{11}, \tilde{\sigma}_{22}, \tilde{\sigma}_{33}, \tilde{\sigma}_{12}, \tilde{\sigma}_{13}\right)$. We can construct $\tilde{\sigma}$ from $\hat{v}=-\left(\Xi_{5}^{-1} v\right)$ :

$$
\tilde{\sigma}=\left(\begin{array}{ccc}
\hat{v}_{1} & \hat{v}_{4} & \hat{v}_{5} \\
\hat{v}_{4} & \hat{v}_{2} & 1 \\
\hat{v}_{5} & 1 & \hat{v}_{3}
\end{array}\right) \tilde{\sigma}_{23}
$$

Then

$$
\begin{aligned}
\operatorname{Det}[\tilde{\sigma}]= & \frac{\operatorname{Det}[\Xi] N_{5}(\tilde{\chi})}{D_{5}(\tilde{\chi})} \\
N_{5}(\tilde{\chi})= & \left(\tilde{\chi}_{13}^{2} \tilde{\chi}_{2}-2 \tilde{\chi}_{12} \tilde{\chi}_{13} \tilde{\chi}_{23}+\tilde{\chi}_{1} \tilde{\chi}_{23}^{2}+\tilde{\chi}_{12}^{2} \tilde{\chi}_{3}-\tilde{\chi}_{1} \tilde{\chi}_{2} \tilde{\chi}_{3}\right)\left(\tilde{\chi}_{12} \tilde{\chi}_{13}^{2}+\tilde{\chi}_{13} \tilde{\chi}_{2} \tilde{\chi}_{23}-\tilde{\chi}_{1} \tilde{\chi}_{12} \tilde{\chi}_{3}\right. \\
& \left.-\tilde{\chi}_{12} \tilde{\chi}_{2} \tilde{\chi}_{3}\right)\left(-\tilde{\chi}_{12}^{2} \tilde{\chi}_{13}+\tilde{\chi}_{1} \tilde{\chi}_{13} \tilde{\chi}_{2}+\tilde{\chi}_{13} \tilde{\chi}_{2} \tilde{\chi}_{3}-\tilde{\chi}_{12} \tilde{\chi}_{23} \tilde{\chi}_{3}\right) \tilde{\sigma}_{23}^{3} \\
D_{5}(\tilde{\chi})= & \left(-\tilde{\chi}_{1} \tilde{\chi}_{12}^{2} \tilde{\chi}_{13}^{2}+\tilde{\chi}_{1}^{2} \tilde{\chi}_{13}^{2} \tilde{\chi}_{2}-\tilde{\chi}_{12}^{2} \tilde{\chi}_{13}^{2} \tilde{\chi}_{2}+\tilde{\chi}_{1} \tilde{\chi}_{13}^{2} \tilde{\chi}_{2}^{2}+\tilde{\chi}_{1}^{2} \tilde{\chi}_{12}^{2} \tilde{\chi}_{3}\right. \\
& -\tilde{\chi}_{12}^{2} \tilde{\chi}_{13}^{2} \tilde{\chi}_{3}-\tilde{\chi}_{1}^{3} \tilde{\chi}_{2} \tilde{\chi}_{3}+\tilde{\chi}_{1} \tilde{\chi}_{12}^{2} \tilde{\chi}_{2} \tilde{\chi}_{3}+\tilde{\chi}_{1} \tilde{\chi}_{13}^{2} \tilde{\chi}_{2} \tilde{\chi}_{3}-\tilde{\chi}_{1}^{2} \tilde{\chi}_{2}^{2} \tilde{\chi}_{3}+\tilde{\chi}_{13}^{2} \tilde{\chi}_{2}^{2} \tilde{\chi}_{3} \\
& -2 \tilde{\chi}_{12} \tilde{\chi}_{13} \tilde{\chi}_{2} \tilde{\chi}_{23} \tilde{\chi}_{3}+\tilde{\chi}_{1} \tilde{\chi}_{2} \tilde{\chi}_{23}^{2} \tilde{\chi}_{3}+\tilde{\chi}_{1} \tilde{\chi}_{12}^{2} \tilde{\chi}_{3}^{2}-\tilde{\chi}_{1}^{2} \tilde{\chi}_{2} \tilde{\chi}_{3}^{2}+\tilde{\chi}_{12}^{2} \tilde{\chi}_{2} \tilde{\chi}_{3}^{2} \\
& \left.-\tilde{\chi}_{1} \tilde{\chi}_{2}^{2} \tilde{\chi}_{3}^{2}\right)^{3}
\end{aligned}
$$

Thus

$$
\because \quad \operatorname{Det}[\tilde{\sigma}] \sim \operatorname{Det}[\boldsymbol{\Xi}] \Rightarrow \operatorname{Det}[\tilde{\sigma}]=0=\operatorname{Det}\left[M_{\nu^{c}}\right]
$$

Since the Majorana mass matrix $M_{\nu^{c}}$ for right handed neutrinos is generated by the VEV $\tilde{\bar{\sigma}}$, it follows that the spectrum includes one or more light sterile neutrino supermultiplets for which the fermionic parts will get Dirac masses only. Thus, if there is one zero eigenvalue (when the other factors in $\operatorname{Det}[\tilde{\sigma}]$ are neither zero nor singular), one will have two superheavy and one zero Majorana mass for the right-handed neutrinos. The two superheavy ones should be integrated out to give a $4 \times 4$ mass matrix for the light neutrinos. Similarly, in the case of two zero eigenvalues, one would integrate out just one right-handed neutrino(see below). 
Of course the mixing structure is then more complicated and must be studied case by case. These are still very interesting cases phenomenologically and it is remarkable that besides the standard three light Majorana neutrinos scenario typical to the NMSGUT, YUMGUTs also throw up scenarios with mixed Majorana-Dirac masses.

One proceeds to solve the $\operatorname{Det}[\boldsymbol{\Xi}]=0$ condition. The prima facie simpler possibility is

$$
\tilde{\chi}_{1}=\frac{\left(\tilde{\chi}_{13}^{2} \tilde{\chi}_{2}-2 \tilde{\chi}_{12} \tilde{\chi}_{13} \tilde{\chi}_{23}+\tilde{\chi}_{12}^{2} \tilde{\chi}_{3}\right)}{\left(\tilde{\chi}_{2} \tilde{\chi}_{3}\right)-\tilde{\chi}_{23}^{2}}
$$

We know $\tilde{\sigma}^{\prime} s$ in terms of $\tilde{\sigma}_{23}$ (Eq.(26) $)$. As in the $N_{g}=2$ case, we calculated $\tilde{\sigma}_{23}$ from one of the equations of Eq.(17) and $P$ completely using Eq.(14). We then solved remaining equations for $A, W$ using a search program.

In the example solution given in the Appendix A, the $\tilde{\sigma} \mathrm{VEV}$ has two zero eigenvalues because the $\operatorname{Det}[\boldsymbol{\Xi}]$ factor that vanishes

$$
\left(\tilde{\chi}_{1} \tilde{\chi}_{2} \tilde{\chi}_{3}-\tilde{\chi}_{1} \tilde{\chi}_{23}^{2}-\tilde{\chi}_{12}^{2} \tilde{\chi}_{3}+2 \tilde{\chi}_{12} \tilde{\chi}_{13} \tilde{\chi}_{23}-\tilde{\chi}_{13}^{2} \tilde{\chi}_{2}\right)
$$

actually occurs twice in $\operatorname{Det}[\tilde{\sigma}]$. We need to integrate out one heavy right handed neutrino

$$
W_{l e p}=\bar{\nu}_{A}^{T} Y_{A B}^{\nu} \nu_{B}+\frac{1}{2} \bar{\nu}_{A}^{T} M_{A B}^{\bar{\nu}} \bar{\nu}_{B}
$$

where $A, B=1,2,3$ and $a, b=1,2$.

$$
\bar{\nu}_{3}=-\frac{Y_{3 A}^{\nu} \nu_{A}}{M_{33}^{\bar{\nu}}}
$$

Effective superpotential is given by (in a diagonal right-handed neutrino basis)

$$
W_{e f f}=\bar{\nu}_{a}^{T} Y_{a B}^{\nu} \nu_{B}+\frac{1}{2} \bar{\nu}_{a}^{T} M_{a a} \bar{\nu}_{a}-\nu_{A}^{T}\left(\frac{1}{2} \frac{Y_{3 A}^{\nu} Y_{3 B}^{\nu}}{M_{33}^{\bar{\nu}}}\right) \nu_{B}
$$

Reading off the light neutrino mass matrix components, we get

$$
\begin{gathered}
\kappa_{A B}=-\left(Y^{\nu}\right)_{A 3}^{T} M_{33}^{-1}\left(Y^{\nu}\right)_{3 B} \\
M_{\text {light }}=\frac{1}{2}\left(\begin{array}{ccccc}
\kappa_{11} & \kappa_{12} & \kappa_{13} & Y_{11}^{\nu} & Y_{21}^{\nu} \\
\kappa_{21} & \kappa_{22} & \kappa_{23} & Y_{12}^{\nu} & Y_{22}^{\nu} \\
\kappa_{31} & \kappa_{32} & \kappa_{33} & Y_{13}^{\nu} & Y_{23}^{\nu} \\
Y_{11}^{\nu} & Y_{12}^{\nu} & Y_{13}^{\nu} & 0 & 0 \\
Y_{21}^{\nu} & Y_{22}^{\nu} & Y_{23}^{\nu} & 0 & 0
\end{array}\right)
\end{gathered}
$$


The doublet Higgs mass matrix becomes 24 dimensional and can be written using $\Omega_{3}[V]$ which can be determined as $\Omega_{2}$ was in Eq. (3). One gets

$$
\Omega_{3}[V]=\left(\begin{array}{cccccc}
V_{11} & 0 & 0 & \frac{V_{12}}{\sqrt{2}} & \frac{V_{13}}{\sqrt{2}} & 0 \\
0 & V_{22} & 0 & \frac{V_{12}}{\sqrt{2}} & 0 & \frac{V_{23}}{\sqrt{2}} \\
0 & 0 & V_{33} & 0 & \frac{V_{13}}{\sqrt{2}} & \frac{V_{23}}{\sqrt{2}} \\
\frac{V_{12}}{\sqrt{2}} & \frac{V_{12}}{\sqrt{2}} & 0 & \frac{V_{11}+V_{22}}{2} & \frac{V_{23}}{2} & \frac{V_{13}}{2} \\
\frac{V_{13}}{\sqrt{2}} & 0 & \frac{V_{13}}{\sqrt{2}} & \frac{V_{23}}{2} & \frac{V_{11}+V_{33}}{2} & \frac{V_{12}}{2} \\
0 & \frac{V_{23}}{\sqrt{2}} & \frac{V_{23}}{\sqrt{2}} & \frac{V_{13}}{2} & \frac{V_{12}}{2} & \frac{V_{22}+V_{33}}{2}
\end{array}\right)
$$

The formulas for the Yukawas are now simple extensions of the $N_{g}=2$ case :

$$
\begin{aligned}
& Y_{u}=\left(\begin{array}{ccc}
\hat{h} \hat{V}_{1}+\hat{f} \hat{V}_{7} & \left(\hat{h} \hat{V}_{4}+\hat{f} \hat{V}_{10}\right) / \sqrt{2} & \left(\hat{h} \hat{V}_{5}+\hat{f} \hat{V}_{11}\right) / \sqrt{2} \\
\left(\hat{h} \hat{V}_{4}+\hat{f} \hat{V}_{10}\right) / \sqrt{2} & \hat{h} \hat{V}_{2}+\hat{f} \hat{V}_{8} & \left(\hat{h} \hat{V}_{6}+\hat{f} \hat{V}_{12}\right) / \sqrt{2} \\
\left(\hat{h} \hat{V}_{5}+\hat{f} \hat{V}_{11}\right) / \sqrt{2} & \left(\hat{h} \hat{V}_{6}+\hat{f} \hat{V}_{12}\right) / \sqrt{2} & \hat{h} \hat{V}_{3}+\hat{f} \hat{V}_{9}
\end{array}\right) \\
& Y_{d}=\left(\begin{array}{ccc}
\hat{h} \hat{W}_{1}+\hat{f} \hat{W}_{13} & \left(\hat{h} \hat{W}_{4}+\hat{f} \hat{W}_{16}\right) / \sqrt{2} & \left(\hat{h} \hat{W}_{5}+\hat{f} \hat{W}_{17}\right) / \sqrt{2} \\
\left(\hat{h} \hat{W}_{4}+\hat{f} \hat{W}_{16}\right) / \sqrt{2} & \hat{h} \hat{W}_{2}+\hat{f} \hat{W}_{14} & \left(\hat{h} \hat{W}_{6}+\hat{f} \hat{W}_{18}\right) / \sqrt{2} \\
\left(\hat{h} \hat{W}_{5}+\hat{f} \hat{W}_{17}\right) / \sqrt{2} & \left(\hat{h} \hat{W}_{6}+\hat{f} \hat{W}_{18}\right) / \sqrt{2} & \hat{h} \hat{W}_{3}+\hat{f} \hat{W}_{15}
\end{array}\right)
\end{aligned}
$$

The Yukawa couplings, mixing angles and neutrino masses for an example solution in this case are exhibited in Table IV, It is interesting that in this case, four of the neutrino masses are in the desirable range of $10^{1 \div 2} \mathrm{meV}$ even though $Y_{u} \neq Y_{\nu}, Y_{d} \neq Y_{l}$. Effectively, the two vanishing $M_{\nu^{c}}$ eigenvalues combined with the mixing of $\nu$ and $\nu^{c}$ via Yukawa couplings gives five light neutrinos of which at least two have significant light sterile admixture. However, such non-minimal scenarios are not yet firmly excluded by the data. The mixing angles can be extracted much as in the case without the neutrino, but the phenomenology is more subtle. The admixture of light sterile neutrinos to obtain neutrino masses in the observed (meV) range indicates a possible route-not eliminated in Refs. [5, 22]- also for the MSGUT by which the no go proved for neutrino masses in the MSGUT might be evaded. In Tables V] and VI, we exhibit the GUT spectra including three singlet pseudo-Goldstones.

\section{2. $\operatorname{Rank}[\boldsymbol{\Xi}]=4$}

In this case, all the $5 \times 5$ minors of $\boldsymbol{\Xi}$ should also vanish along with $\operatorname{Det}[\boldsymbol{\Xi}]$. One can determine four $\tilde{\sigma}^{\prime} s$ in terms of the remaining two. Suppose we calculate $\tilde{\sigma}_{11}, \tilde{\sigma}_{22}, \tilde{\sigma}_{33}$ and 
$\tilde{\sigma}_{12}$ in terms of $\left(\tilde{\sigma}_{13}, \tilde{\sigma}_{23}\right)$. Then

$$
\tilde{\sigma}=A \tilde{\sigma}_{13}+B \tilde{\sigma}_{23}
$$

where the matrices $\mathrm{A}$ and $\mathrm{B}$ are functions of the $\tilde{\chi}_{A B}$. Now, $\operatorname{Det}[\tilde{\sigma}]$ factors will involve $\tilde{\sigma}_{13}$, $\tilde{\sigma}_{23}$ instead of involving purely $\tilde{\chi}$ elements with the undetermined $\tilde{\sigma}$ component factorizing outside as in case of $\operatorname{Rank}[\boldsymbol{\Xi}]=5$. Even if one assumes $\tilde{\sigma}_{13}=\tilde{\sigma}_{23}$ (although there is no reason to assume this), we find that $\operatorname{Det}[\tilde{\sigma}]$ still has no common factor with $\operatorname{Det}[\boldsymbol{\Xi}]$. So $\operatorname{Rank}[\boldsymbol{\Xi}]=4$ could be a workable scenario with type I seesaw neutrino masses and without light sterile neutrinos. Now, we discuss the conditions for vanishing minors.

All the $5 \times 5$ minors have one common factor. Ideally, one can solve the equations by putting the common factor of the minors equal to zero but the resulting system becomes very complicated. For simplicity, we equate to zero two factors from the dimension-5 minors the vanishing of which implies the vanishing of all the dimension-5 minors. In all, we get three conditions, one from $\operatorname{Det}[\boldsymbol{\Xi}]=0$ (Eq. 24 ) and two from $5 \times 5$ minors.

Minors of order 5 are null if we require

$$
\begin{array}{r}
\tilde{\chi}_{13} \tilde{\chi}_{23}-\tilde{\chi}_{12} \tilde{\chi}_{3}=0 \\
\tilde{\chi}_{12}^{2} \tilde{\chi}_{13}+\tilde{\chi}_{12} \tilde{\chi}_{2} \tilde{\chi}_{23}+\tilde{\chi}_{13} \tilde{\chi}_{23}^{2}=0
\end{array}
$$

We solve the above equations for $\tilde{\chi}_{2}$ and $\tilde{\chi}_{3}$ :

$$
\tilde{\chi}_{1}=\frac{\tilde{\chi}_{12} \tilde{\chi}_{13}}{\tilde{\chi}_{23}} ; \quad \tilde{\chi}_{2}=\frac{-\tilde{\chi}_{13}\left(\tilde{\chi}_{12}^{2}+\tilde{\chi}_{23}^{2}\right)}{\tilde{\chi}_{12} \tilde{\chi}_{23}} \quad ; \quad \tilde{\chi}_{3}=\frac{\tilde{\chi}_{13} \tilde{\chi}_{23}}{\tilde{\chi}_{12}}
$$

Now we have four $\tilde{\sigma}$ equations along with above three conditions. We used the third extra condition to fix the $\xi$ parameter to maintain the consistency of the system of equations. Therefore, $\xi$ is not a random parameter but is fixed in terms of $\operatorname{VEVs}(P, A, W)$. The alternatives to this will be examined elsewhere.

We calculate the eigenvalues of SM fermions and neutrino Yukawas (Eq. (36)) along with quark and lepton mixing angles for large and small values of $f$ and a random illustrative set of superpotential parameters. The results are given as Tables VII and VIII.

As in the $N_{g}=2$ case, observe that if $f$ affects the charged fermion Yukawas significantly, avoiding $Y_{u}=Y_{\nu}, Y_{d}=Y_{l}$, and generates appreciable mixing then the type I seesaw neutrino masses are much too small. While if we boost the type I seesaw masses by lowering $f, Y_{\nu} \simeq$ $Y_{u}, Y_{l} \simeq Y_{d}$ and Cabibbo Kobayashi Maskawa(CKM) angles are negligible. The complete 
superheavy spectra are given in Tables $[\mathrm{X}]$ and $\mathrm{X}$, and again we have (4) standard model singlet pseudo-Goldstones.

\section{Traceless Symmetric multiplets of $\mathrm{O}(3)$}

As we saw earlier, the (reducible) six-dimensional symmetric representation of $\mathrm{O}(3)$ with equal superpotential couplings for traceless(5-plet) and singlet part led, in the nondegenerate case $(\operatorname{Rank}[\boldsymbol{\Xi}]=5)$ to a light sterile neutrino(s). Another alternative is to utilize only traceless representations in the (visible sector) GUT so that $P, A, W, \tilde{\sigma}, \tilde{\bar{\sigma}}$ are all traceless symmetric matrices. This reduces the dimension and rank of the homogeneous system $(\boldsymbol{\Xi} \cdot \hat{\boldsymbol{\Sigma}}=\mathbf{0})$ and makes it possible to find non-singular $\tilde{\sigma}(\tilde{\bar{\sigma}})$ VEVs thus avoiding light sterile neutrinos. We write the traceless symmetric $3 \times 3$ representation as

$$
\hat{\phi}_{A B}=\phi_{11} \frac{\left(\lambda_{3}\right)_{A B}}{\sqrt{2}}+\phi_{22} \frac{\left(\lambda_{8}\right)_{A B}}{\sqrt{2}}+\frac{\phi_{K L}}{\sqrt{2}} \delta_{(A}^{K} \delta_{B)}^{L}
$$

Here, $\lambda_{3}$ and $\lambda_{8}$ are the usual diagonal $3 \times 3$ Gell-Mann matrices. Matrix $\boldsymbol{\Xi}$ is given by

$$
\boldsymbol{\Xi}=\left(\begin{array}{ccccc}
-\tilde{\chi}_{22}+2 \xi & -\tilde{\chi}_{11}-\tilde{\chi}_{22}+\xi & \tilde{\chi}_{12} & 0 & -\tilde{\chi}_{23} \\
-\tilde{\chi}_{11}-\tilde{\chi}_{22}+\xi & -\tilde{\chi}_{11}+2 \xi & \tilde{\chi}_{12} & -\tilde{\chi}_{13} & 0 \\
\frac{\tilde{\chi}_{12}}{2} & \frac{\tilde{\chi}_{12}}{2} & \frac{\left(\tilde{\chi}_{11}+\tilde{\chi}_{22}+2 \xi\right)}{2} & \frac{\tilde{\chi}_{23}}{2} & \frac{\tilde{\chi}_{13}}{2} \\
0 & -\frac{\tilde{\chi}_{13}}{2} & \frac{\tilde{\chi}_{23}}{2} & \frac{-\tilde{\chi}_{22}+2 \xi}{2} & \frac{\tilde{\chi}_{12}}{2} \\
-\frac{\tilde{\chi}_{23}}{2} & 0 & \frac{\tilde{\chi}_{13}}{2} & \frac{\tilde{\chi}_{12}}{2} & \frac{-\tilde{\chi}_{11}+2 \xi}{2}
\end{array}\right)
$$

$\hat{\Sigma}=\left\{\tilde{\sigma}_{11}, \tilde{\sigma}_{22}, \tilde{\sigma}_{12}, \tilde{\sigma}_{13}, \tilde{\sigma}_{23}\right\}$. As discussed above it is useful to define a matrix function $(\Omega[V]$ ) to write the mass matrices in compact notation and is specified by the symmetric invariant for the singlet product of three traceless symmetric irreps. In the present scenario, it has the following form :

$$
\Omega_{3}^{\prime}[V]=\left(\begin{array}{ccccc}
\frac{V_{11}+V_{22}}{2} & \frac{V_{11}-V_{22}}{2 \sqrt{3}} & 0 & \frac{V_{13}}{2} & -\frac{V_{23}}{2} \\
\frac{V_{11}-V_{22}}{2 \sqrt{3}} & \frac{-V_{11}-V_{22}}{2} & \frac{V_{12}}{\sqrt{3}} & -\frac{V_{13}}{2 \sqrt{3}} & -\frac{V_{23}}{2 \sqrt{3}} \\
0 & \frac{V_{12}}{\sqrt{3}} & \frac{V_{11}+V_{22}}{2} & \frac{V_{23}}{2} & \frac{V_{13}}{2} \\
\frac{V_{13}}{2} & -\frac{V_{13}}{2 \sqrt{3}} & \frac{V_{23}}{2} & -\frac{V_{22}}{2} & \frac{V_{12}}{2} \\
-\frac{V_{23}}{2} & -\frac{V_{23}}{2 \sqrt{3}} & \frac{V_{13}}{2} & \frac{V_{12}}{2} & -\frac{V_{11}}{2}
\end{array}\right)
$$

Rows and columns of the Higgs mass matrix are labelled by $\left\{\frac{\left(\bar{H}_{11}-\bar{H}_{22}\right)}{\sqrt{2}}, \sqrt{\frac{3}{2}}\left(\bar{H}_{11}+\right.\right.$ $\left.\left.\bar{H}_{22}\right), \sqrt{2} \bar{H}_{12}, \sqrt{2} \bar{H}_{13}, \sqrt{2} \bar{H}_{23}\right\}$ and $\left\{\frac{\left(H_{11}-H_{22}\right)}{\sqrt{2}}, \sqrt{\frac{3}{2}}\left(H_{11}+H_{22}\right), \sqrt{2} H_{12}, \sqrt{2} H_{13}, \sqrt{2} H_{23}\right\}$. Up 
and down quark Yukawas are given as :

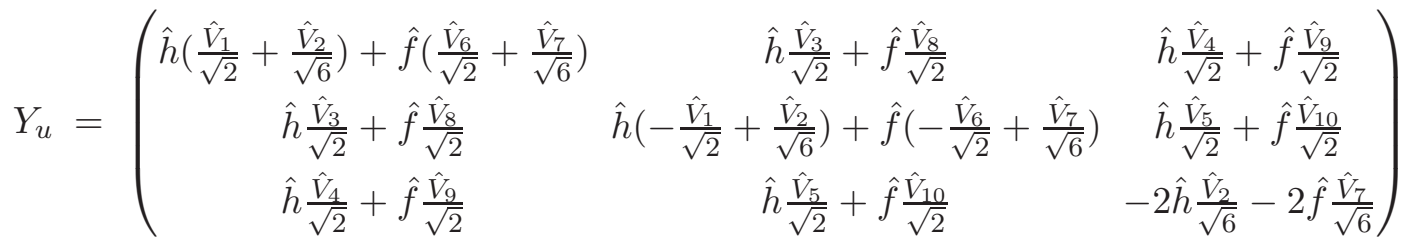

$$
\begin{aligned}
& Y_{d}=\left(\begin{array}{ccc}
\hat{h}\left(\frac{\hat{W}_{1}}{\sqrt{2}}+\frac{\hat{W}_{2}}{\sqrt{6}}\right)+\hat{f}\left(\frac{\hat{W}_{11}}{\sqrt{2}}+\frac{\hat{W}_{12}}{\sqrt{6}}\right) & \hat{h} \frac{\hat{W}_{3}}{\sqrt{2}}+\hat{f} \frac{\hat{W}_{13}}{\sqrt{2}} & \hat{h} \frac{\hat{W}_{4}}{\sqrt{2}}+\hat{f} \frac{\hat{W}_{14}}{\sqrt{2}} \\
\hat{h} \frac{\hat{W}_{3}}{\sqrt{2}}+\hat{f} \frac{\hat{W}_{13}}{\sqrt{2}} & \hat{h}\left(-\frac{\hat{W}_{1}}{\sqrt{2}}+\frac{\hat{W}_{2}}{\sqrt{6}}\right)+\hat{f}\left(-\frac{\hat{W}_{11}}{\sqrt{2}}+\frac{\hat{W}_{12}}{\sqrt{6}}\right) & \hat{h} \frac{\hat{W}_{5}}{\sqrt{2}}+\hat{f} \frac{\hat{W}_{15}}{\sqrt{2}} \\
\hat{h} \frac{\hat{W}_{4}}{\sqrt{2}}+\hat{f} \frac{\hat{W}_{14}}{\sqrt{2}} & \hat{h} \frac{\hat{W}_{5}}{\sqrt{2}}+\hat{f} \frac{\hat{W} 15}{\sqrt{2}} & -2 \hat{h} \frac{\hat{W}_{2}}{\sqrt{6}}-2 \hat{f} \frac{\hat{W}_{12}}{\sqrt{6}}
\end{array}\right)
\end{aligned}
$$

We have solved the least degenerate $(\operatorname{Rank}[\boldsymbol{\Xi}]=4)$ case. Yukawa eigenvalues and mixing angles are given in Tables XI.

The complete mass spectra are given in Tables XII and XIII. There are three MSSM singlet pseudo-Goldstone supermultiplets left behind as a relic of the GUT and flavour symmetry breaking.

From Tables VII and XI, we conclude that it is possible to fit the charged fermion and neutrino mixing data in the case without the 120-plet without light sterile neutrinos but that the neutrino masses are too small. Raising the neutrino masses requires that an additional multiplet is required to contribute to fit the charged fermion masses and mixing : the obvious choice if duplication of Higgs is to be avoided is the 120-plet as in the case of the NMSGUT. If we allow light sterile neutrinos (as we saw for $N_{g}=3 \operatorname{Rank}[\boldsymbol{\Xi}]=5$ ) then we can even get adequate neutrino masses without $Y_{u}=Y_{\nu}, Y_{d}=Y_{l}$ and possibly viable phenomenology. This needs to be studied.

\section{DISCUSSION}

In this paper, we have proposed a gauge unification of flavour in tandem with the realistic grand unification achieved in the context of the NMSGUT [4 7, 10]. The flavour symmetry is broken along with $\mathrm{SO}(10)$ gauge symmetry at the GUT scale by the same multiplets that break GUT symmetry. However, a special (Bajc-Melfo) hidden sector was introduced[15] to allow supersymmetry preservation by the VEV of the flavour group D-terms. The central idea exploited for generating flavour structure in the effective theory is the non-linear percolation of flavour breaking effects through the imposition of the phenomenological constraint that a single pair of light doublets emerges in the effective MSSM from the multiple doublets in the GUT. The consistent treatment of the implications of this constraint had 
already paid[7] rich dividends such as a solution of the perennial problem of fast dimension 5 operator proton decay in SUSY GUTs. This resolution has driven home the message that careful attention to the existence conditions for the light Higgs opens a portal into the entrails of complicated UV completions precisely because light MSSM Higgs arise as mixtures of doublets from multiple sources in the GUT Higgs set and are thus connected to the entire UV completion by non-gauge interactions : in contrast to the rest of the fields of the MSSM. In view of the understanding conferred, we call such flavour "tasty flavour" and these models Yukawon ultraminimal GUTs. This mechanism, like that [7] for suppressing $d=5 \mathrm{~B}$ violation in the NMSGUT, depends on enforcing consistency conditions between a single light pair of MSSM doublets and the multiple doublets in the full theory. A common apprehension is that pseudo-Goldstone multiplets may arise due to the duplication of Higgs multiplets under family symmetry. However, we have checked the complete spectra in the GUT sector and only present those solutions without pseudo-Goldstone multiplets that ruin unification (which do occur in certain degenerate cases). The values of the fields and complete associated spectra for the example solutions are given in Appendix B. This shows that the appearance of pseudo-Goldstones is not inevitable except the SM singlet sector which does not effect unification and moreover furnishes dark matter candidates or otherwise constrains the flavour symmetry introduced.

Therefore, we expect that there should exist regions of the (much reduced relative to MSGUTs) flavour bland parameter space of YUMGUTs that generate actual MSSM Yukawas. Determination of GUT parameter values that generate the observed set of Yukawa couplings, symmetry breaking, neutrino masses, B-decay and other exotic phenomenon rates requires an elaborate investigation using a generalization of computer codes by which we obtained completely realistic fits of all fermion data and distinctive predictions for SUSY spectra in the context of the NMSGUT[6]. This will be reported in sequels. In this paper, we have shown how even random values of the YUMGUT parameters can generate a fermion hierarchy at least qualitatively similar to the one actually observed. An important point that emerges is that an effective theory with light sterile neutrinos can emerge naturally from the spontaneous symmetry breaking so that even scenarios with relatively large values of $f$ may lead to a viable phenomenology. This discovery underlines the need to reinvestigate the MSGUT to explore the possibility that it may still yield acceptable neutrino masses by giving ultralight sterile neutrinos mixed through Yukawa couplings with active neutrinos( 
which are therefore heavy enough) even when the $\overline{\mathbf{1 2 6}}$ coupling is large enough to contribute significantly to the charged fermion masses and thus type I masses are negligible. This possibility had been overlooked earlier [5, 22, 23]. Of course, the other possibility is that an acceptable low-energy theory arises by mimicking the NMSGUT fit i.e 10,120 couplings responsible for charged fermion masses and small $f,<\overline{\mathbf{1 2 6}}>$ give right-handed neutrinos light enough to give acceptable type I seesaw masses for three light left-handed neutrinos and no sterile neutrinos.

A striking feature of the model is the ubiquity of MSSM singlet pseudo-Goldstones from the GUT symmetry breaking which occur in each one of the models studied. In some sense, these are "moduli" multiplets familiar in other contexts, such as string theory, which generate families. They may serve as a welcome signal of, and constraint on, these models. Soft SUSY breaking and radiative corrections to their masses and the associated phenomenology and cosmological implications deserve detailed investigation.

To summarize, R-parity preserving minimal SUSY SO(10) theories offer a radical conceptual simplification that melds the problems of the fermion flavour hierarchy and GUT symmetry breaking in a novel way. Innovation arises from a very topical emphasis on how the Higgs portal opens into the UV completion of the MSSM. Our model for family and GUT unification is based on the conviction that, as once promised by string theory, it is the number of couplings, not the number of fields, that should be minimized. $\mathrm{SO}(10)$ "Yukawonification" is concrete, calculable (at least for (N)MSGUTs where much of the tedious but directly useable (see e.g. Eq.(22) preparatory work on group theory[11] and mass spectra [6, 10 12, 24] is already done) and falsifiable. Since $O\left(N_{g}\right)$ contains most of the commonly fancied discrete groups, even discrete group model builders may benefit from the structural hints provided by $S O(10) \times O\left(N_{g}\right)$ models along with a welcome calculability as regards the "vacuum alignment" : specified here by parameters that determine both the GUT SSB and MSSM mass-mixing data. 


\section{APPENDIX A : YUMGUT SUPERHEAVY VEVS}

In this Appendix we give solutions of the spontaneous symmetry breaking in the toy $\left(N_{g}=2\right)$ and realistic $\left(N_{g}=3\right)$ cases.

A. $\quad N_{g}=\mathbf{2}, \operatorname{Rank}[\boldsymbol{\Xi}]=\mathbf{2}$

The values of the VEVs of the YUMGUT Higgs fields responsible for breaking $S O(10) \rightarrow$ MSSM in units of $m / \lambda \sim 10^{16} \mathrm{GeV}$ are

$$
\begin{aligned}
W & =\left(\begin{array}{cc}
0.141-0.203 i & 0.3168+0.189 i \\
0.3168+0.189 i & -0.2667+0.3075 i
\end{array}\right) \quad P=\left(\begin{array}{cc}
-0.236-0.2001 i & 0.1787-0.028 i \\
0.1787-0.028 i & -0.2297+0.1202 i
\end{array}\right) \\
A & =\left(\begin{array}{cc}
-0.23-0.3521 i & 0.3382+0.0777 i \\
0.3382+0.0777 i & -0.4475+0.2227 i
\end{array}\right) \quad \tilde{\sigma}=\tilde{\bar{\sigma}}=\left(\begin{array}{cc}
0.0863-0.2366 i & 0.1973+0.1041 i \\
0.1973+0.1041 i & -0.0863+0.2366 i
\end{array}\right) \\
\bar{D}_{X}= & 2\left(\left|p_{+}\right|^{2}-\left|p_{-}\right|^{2}+3\left(\left|a_{+}\right|^{2}-\left|a_{-}\right|^{2}\right)+6\left(\left|w_{+}\right|^{2}-\left|w_{-}\right|^{2}\right)+\frac{1}{2}\left|\sigma_{+}\right|^{2}-\left|\sigma_{-}\right|^{2}+\frac{1}{2}\left|\bar{\sigma}_{+}\right|^{2}-\left|\bar{\sigma}_{-}\right|^{2}\right) \\
& =-8.94
\end{aligned}
$$

\section{B. $N_{g}=3$}

As discussed in [13] traceless part of BM field $S_{a b}$ is used to cancel $O\left(N_{g}\right)$ D-terms. For

fixing $\hat{S}_{a b}$, without loss of generality, one can rotate the fields by an $O(3)$ transformation so that the so that only one component of the D-term vector is non zero. VEVs below are written in the basis where only the third D-term component $<D^{3}>$ is non zero. This basis is denoted by a prime on all VEVs. 
1. $\operatorname{Rank}[\boldsymbol{\Xi}]=5$ (tracefull symmetric representation)

$$
\begin{aligned}
\tilde{\sigma}^{\prime}=\tilde{\bar{\sigma}}^{\prime}= & \left(\begin{array}{ccc}
-8.1532+14.4793 i & -11.7404-7.4196 i & 0.194-1.1043 i \\
-11.7404-7.4196 i & 6.6912-9.4853 i & 0.9135+0.2088 i \\
0.194-1.1043 i & 0.9135+0.2088 i & 0.0124+0.0746 i
\end{array}\right) \\
A^{\prime}= & \left(\begin{array}{ccc}
-2.7937-0.4459 i & -0.0298-1.8182 i & 2.573-0.2572 i \\
-0.0298-1.8182 i & -2.6651+0.5903 i & -0.4297-1.5744 i \\
2.573-0.2572 i & -0.4297-1.5744 i & -0.8325-0.1193 i
\end{array}\right) \\
P^{\prime}= & \left(\begin{array}{ccc}
2.6802-0.9855 i & 0.589-1.518 i & -13.9797+0.005 i \\
0.589-1.518 i & 4.1178+0.5751 i & 1.9362-0.8664 i \\
-13.9797+0.005 i & 1.9362-0.8664 i & 6.1195+0.1825 i
\end{array}\right) \\
W^{\prime}= & \left(\begin{array}{ccc}
1.0342+0.1527 i & -0.4614+0.2617 i & 2.0891+0.4169 i \\
-0.4614+0.2617 i & -1.4776-0.1941 i & -0.3529+2.8359 i \\
2.0891+0.4169 i & -0.3529+2.8359 i & 1.3112+0.0711 i
\end{array}\right) \\
D_{X}^{\prime a}=3319.0 \delta_{3}^{a} &
\end{aligned}
$$

2. $\operatorname{Rank}[\boldsymbol{\Xi}]=4$ (tracefull symmetric representation)

$$
\begin{gathered}
\tilde{\sigma}^{\prime}=\tilde{\bar{\sigma}}^{\prime}=\left(\begin{array}{ccc}
-0.0515-2.2441 i & 1.6389+0.9556 i & 0.8735+1.689 i \\
1.6389+0.9556 i & 0.4307+0.2916 i & -1.8341-2.1534 i \\
0.8735+1.689 i & -1.8341-2.1534 i & 0.926+1.8694 i
\end{array}\right) \\
A^{\prime}=\left(\begin{array}{ccc}
-1.1162+0.1493 i & -0.6261-0.4133 i & -0.1611+0.4422 i \\
-0.6261-0.4133 i & 0.3311+0.7292 i & -0.3128-0.1764 i \\
-0.1611+0.4422 i & -0.3128-0.1764 i & -0.7771-0.6807 i
\end{array}\right)
\end{gathered}
$$




$$
\begin{gathered}
P^{\prime}=\left(\begin{array}{ccc}
0.7956-0.2474 i & 0.0406-0.4419 i & 0.6712+0.2745 i \\
0.0406-0.4419 i & 0.3486+1.7078 i & -0.0373-0.1994 i \\
0.6712+0.2745 i & -0.0373-0.1994 i & -0.4144-0.7517 i
\end{array}\right) \\
W^{\prime}=\left(\begin{array}{ccc}
-0.0025-0.4259 i & 0.0644+0.4904 i & -0.3268-0.5708 i \\
0.0644+0.4904 i & -0.3575-0.0647 i & 0.0554+0.2052 i \\
-0.3268-0.5708 i & 0.0554+0.2052 i & 0.5644+0.6209 i
\end{array}\right) \\
D_{X}^{\prime a}=47.04 \delta_{3}^{a}
\end{gathered}
$$

C. $\operatorname{Rank}[\Xi]=4$ (traceless symmetric representation)

$$
\begin{aligned}
\tilde{\sigma}^{\prime}=\tilde{\sigma}^{\prime}= & \left(\begin{array}{ccc}
1.5506-5.4394 i & 1.7398+4.1109 i & 0.0011-0.0811 i \\
1.7398+4.1109 i & -1.6868+2.0758 i & 0.0202+0.042 i \\
0.0011-0.0811 i & 0.0202+0.042 i & 0.1361+3.3636 i
\end{array}\right) \\
A^{\prime}= & \left(\begin{array}{ccc}
-1.8304-0.7199 i & 1.5213-0.445 i & -0.0854+0.0056 i \\
1.5213-0.445 i & 0.6854+0.7288 i & -0.0906-0.0529 i \\
-0.0854+0.0056 i & -0.0906-0.0529 i & 1.145-0.009 i
\end{array}\right) \\
P^{\prime}= & \left(\begin{array}{rrr}
-0.2976+0.0331 i & 0.3609-0.0673 i & -0.2904+0.0343 i \\
0.3609-0.0673 i & 0.2394+0.4181 i & -0.5147-0.2562 i \\
-0.2904+0.0343 i & -0.5147-0.2562 i & 0.0581-0.4512 i
\end{array}\right) \\
W^{\prime}= & \left(\begin{array}{rrr}
1.0622+0.3626 i & -0.7826+0.3279 i & -0.0567+0.0102 i \\
-0.7826+0.3279 i & -0.3667-0.2597 i & -0.1406-0.0597 i \\
-0.0567+0.0102 i & -0.1406-0.0597 i & -0.6955-0.1029 i
\end{array}\right) \\
D_{X}^{\prime a}=275.15 \delta_{3}^{a} &
\end{aligned}
$$

\section{APPENDIX B : ILLUSTRATIVE TABLES OF YUKAWA STRUCTURE AND SUPERHEAVY SPECTRA IN YUMGUTS}

In this Appendix, we give the Yukawa hierarchies and complete superheavy spectra for the three example solutions discussed above, in units of the MSGUT scale parameter $m / \lambda$. 


\begin{tabular}{|c|c|c|c|c|c|}
\hline S.No. & $\mathrm{M}_{\mathrm{H}}$ & $\mathrm{Y}_{\mathrm{u}}$ & $\mathrm{Y}_{\mathrm{d}}$ & $\theta_{\mathrm{CKM}}(\mathrm{deg})$. & $m_{\nu}(\mathrm{meV})$ \\
\hline 1. & $0.049+0.190 i$ & $0.1537,0.0080$ & $0.0537,0.0043$ & 5.15 & $0.0255,0.2791$ \\
2. & $0.599+0.791 i$ & $0.1293,0.0118$ & $0.0562,0.0051$ & $2.27 \times 10^{-6}$ & $0.0013,0.0144$ \\
3. & $1.39+0.80 i$ & $0.0685,0.0214$ & $0.0359,0.0052$ & 7.41 & $0.0011,0.0121$ \\
\hline S.No. & $\mathrm{Y}_{1}$ & $Y_{\nu}$ & $\theta_{\mathrm{PMNS}}(\mathrm{deg})$. & $\tilde{M}_{\nu^{c}}$ & $\Delta m_{\nu}^{2}\left(\mathrm{eV}^{2}\right)$ \\
\hline 1. & $0.0424,0.0027$ & $0.2515,0.0233$ & 14.5 & $0.6967,0.0636$ & $7.73 \times 10^{-8}$ \\
2. & $0.0712,0.0065$ & $0.0576,0.0053$ & $2.32 \times 10^{-5}$ & $0.6969,0.0636$ & $2.06 \times 10^{-10}$ \\
3. & $0.0147,0.0063$ & $0.0911,0.0028$ & 33.7 & $0.6969,0.0636$ & $1.45 \times 10^{-10}$ \\
\hline
\end{tabular}

TABLE I: Yukawa eigenvalues and mixing angles for $N_{g}=2, f=-0.13 \cdot \tilde{M}_{\nu^{c}} \equiv \lambda M_{\nu^{c}} / m . m / \lambda$ is taken to be $10^{16} \mathrm{GeV}$ to estimate $\Delta m_{\nu}^{2} . \lambda=-0.038+.005 i, \eta=0.4, \gamma=0.32, \bar{\gamma}=-1.6, h=.34$, $\xi=0.8719+.5474 i . M_{\nu^{c}}$ is independent of $M_{H}$ value chosen.

\begin{tabular}{|c|c|c|c|c|c|}
\hline S.No. & $\mathrm{M}_{\mathrm{H}}$ & $\mathrm{Y}_{\mathrm{u}}$ & $\mathrm{Y}_{\mathrm{d}}$ & $\theta_{\mathrm{CKM}}(\mathrm{deg})$. & $m_{\nu}(\mathrm{meV})$ \\
\hline 1. & $0.049+0.190 i$ & $0.1761,0.0131$ & $0.0507,0.0038$ & 0.00486 & $10.05,110.19$ \\
2. & $0.599+0.791 i$ & $0.1108,0.0101$ & $0.0569,0.0052$ & $2.47 \times 10^{-9}$ & $4.86,53.29$ \\
3. & $1.39+0.80 i$ & $0.0721,0.0140$ & $0.0283,0.00552$ & 0.00767 & $4.39,48.12$ \\
\hline S.No. & $\mathrm{Y}_{1}$ & $Y_{\nu}$ & $\theta_{\mathrm{PMNS}}($ deg. $)$ & $\tilde{M}_{\nu^{c}}$ & $\Delta m_{\nu}^{2}\left(\mathrm{eV}^{2}\right)$ \\
\hline 1. & $0.0507,0.0038$ & $0.1762,0.0131$ & 8.7 & $0.000697,0.0000636$ & 0.01204 \\
2. & $0.0569,0.0052$ & $0.1108,0.0101$ & $2.79 \times 10^{-6}$ & $0.000697,0.0000636$ & 0.00282 \\
3. & $0.0283,0.0055$ & $0.0721,0.0140$ & 26.8 & $0.000697,0.0000636$ & 0.00230 \\
\hline
\end{tabular}

TABLE II: Effect of reducing $f$ : Yukawa eigenvalues and mixing angles for $N_{g}=2, f=-0.00013$ and other parameters same as in Table【. Notice that the light neutrino masses are in an acceptable range but $Y_{u}=Y_{\nu}, Y_{d}=Y_{l}$ and the quark mixing is negligible.

This is done to remove any prejudice that unacceptable pseudo-Goldstone multiplets must occur in family symmetric models. The naming convention and MSGUT multiplicities can be found in[10]. 


\begin{tabular}{|c|c|c|c|}
\hline $\begin{array}{c}\text { Field } \\
{[\mathrm{SU}(3), \mathrm{SU}(2), \mathrm{Y}]}\end{array}$ & Masses & $\begin{array}{c}\text { Field } \\
{[\mathrm{SU}(3), \mathrm{SU}(2), \mathrm{Y}]}\end{array}$ & Masses \\
\hline$A[1,1,4]$ & $4.093,3.321,0.137$ & $B[6,2,5 / 3]$ & $0.106,0.099,0.091$ \\
\hline$C[8,2,1]$ & $1.727,1.727,1.224$ & $C[8,2,1]$ & $1.224,0.614,0.614$ \\
\hline$D[3,2,7 / 3]$ & $1.919,1.433,1.191$ & $D[3,2,7 / 3]$ & $0.810,0.205,0.134$ \\
\hline$E[3,2,1 / 3]$ & $1.475,1.043,0.716$ & $E[3,2,1 / 3]$ & $0.716,0.677,0.594,0.506$ \\
\hline$E[3,2,1 / 3]$ & $0.404,0.277,0.087$ & $E[3,2,1 / 3]$ & $0.073,0.050,0.004$ \\
\hline$F[1,1,2]$ & $1.794,1.794,1.681$ & $F[1,1,2]$ & $1.317,0.289,0.228,0.018$ \\
\hline$G[1,1,0]$ & $1.672,1.665,1.248$ & $G[1,1,0]$ & $1.248,0.766,0.766,0.504$ \\
\hline$G[1,1,0]$ & $0.469,0.208,0.201$ & $G[1,1,0]$ & $0.079,0.068,0.055$ \\
\hline$G[1,1,0]$ & $0.011,0.009,0$ & $h^{(1)}[1,2,1]$ & $3.799,2.812,1.398,1.182,0.983$ \\
\hline$h^{(1)}[1,2,1]$ & $0.74,0.588,0.511,0.159,0.024,0.013$ & $h^{(2)}[1,2,1]$ & $3.947,2.961,1.623,1.247,1.009$ \\
\hline$h^{(2)}[1,2,1]$ & $0.726,0.556,0.51,0.14,0.044,0.005$ & $h^{(3)}[1,2,1]$ & $4.161,3.196,2.049$ \\
\hline$h^{(3)}[1,2,1]$ & $1.289,0.979,0.710$ & $h^{(3)}[1,2,1]$ & $.540, .520, .152, .029, .010$ \\
\hline$I[3,1,10 / 3]$ & $0.210,0.192,0.003$ & $J[3,1,4 / 3]$ & $1.889,1.889,0.946$ \\
\hline$J[3,1,4 / 3]$ & $0.740,0.453,0.278$ & $J[3,1,4 / 3]$ & $0.119,0.086,0.021,0.006$ \\
\hline$K[3,1,-8 / 3]$ & $1.591,1.237,0.116$ & $L[6,1,2 / 3]$ & $1.066,0.916,0.757$ \\
\hline$M[6,1,8 / 3]$ & $1.340,0.958,0.493$ & $N[6,1,-4 / 3]$ & $1.795,1.178,0.345$ \\
\hline$O[1,3,-2]$ & $1.127,0.886,0.084$ & $P[3,3,-2 / 3]$ & $0.902,0.754,0.595$ \\
\hline$Q[8,3,0]$ & $0.163,0.126,0.083$ & $R[8,1,0]$ & $0.170,0.119,0.107$ \\
\hline$R[8,1,0]$ & $0.086,0.066,0.047$ & $S[1,3,0]$ & $0.090,0.058,0.011$ \\
\hline$t^{(1)}[3,1,-2 / 3]$ & $3.264,2.802,1.824,1.496,1.175$ & $t^{(1)}[3,1,-2 / 3]$ & $1.019,0.89,0.824,0.598,0.495$ \\
\hline$t^{(1)}[3,1,-2 / 3]$ & $0.343,0.202,0.055,0.026,0.007$ & $t^{(2)}[3,1,-2 / 3]$ & $3.418,2.936,1.873,1.636,1.2$ \\
\hline$t^{(2)}[3,1,-2 / 3]$ & $1.053,0.909,0.824,0.692,0.532$ & $t^{(2)}[3,1,-2 / 3]$ & $0.454,0.211,0.077,0.018,0.001$ \\
\hline$t^{(3)}[3,1,-2 / 3]$ & $3.650,3.156,2.097,1.747,1.273$ & $t^{(3)}[3,1,-2 / 3]$ & $1.116,0.926,0.824,0.779,0.541$ \\
\hline$t^{(3)}[3,1,-2 / 3]$ & $0.466,0.223,0.116,0.023,0.002$ & $U[3,3,4 / 3]$ & $0.084,0.070,0.054$ \\
\hline$V[1,2,-3]$ & $0.227,0.208,0.003$ & $W[6,3,2 / 3]$ & $1.693,1.324,0.902$ \\
\hline$X[3,2,-5 / 3]$ & $1.666,1.666,0.149$ & $X[3,2,-5 / 3]$ & $.102, .072, .070, .066$ \\
\hline$Y[6,2,-1 / 3]$ & $0.167,0.118,0.058$ & $Z[8,1,2]$ & $0.100,0.086,0.070$ \\
\hline
\end{tabular}

TABLE III: Mass Spectrum of superheavy fields in units of $m / \lambda \sim 10^{16} \mathrm{GeV}$ for $N_{g}=2$ for the solution described in Section IV.A. Only the spectra of $h[1,2, \pm 1], t[3,1, \mp 2 / 3]$ depend on the three possible values of $M_{H}$ (see Table I for the values of $M_{H}$ ). The corresponding sets are labelled as 


\begin{tabular}{|c|c|c|c|c|}
\hline S.No. & $\mathrm{M}_{\mathrm{H}}$ & $\mathrm{Y}_{\mathrm{u}}$ & $\mathrm{Y}_{\mathrm{d}}$ & $\begin{array}{c}\left\{\theta_{13}, \theta_{12}, \theta_{23}\right\}^{\mathrm{Q}} \\
(\text { deg. })\end{array}$ \\
\hline 1. & $2.55+0.13 i$ & $0.007,0.019,0.368$ & $0.007,0.014,0.306$ & $0.56,13.18,1.58$ \\
2. & $1.44-0.61 i$ & $0.027,0.13,0.409$ & $0.009,0.083,0.242$ & $3.42,8.71,3.87$ \\
3. & $1.28+0.75 i$ & $0.063,0.228,0.424$ & $0.019,0.083,0.186$ & $6.65,6.59,1.11$ \\
4. & $1.16+0.67 i$ & $0.062,0.193,0.439$ & $0.02,0.099,0.188$ & $2.6,5.18,1.96$ \\
5. & $1.06-0.73 i$ & $0.009,0.076,0.458$ & $0.008,0.078,0.321$ & $1.51,11.19,4.61$ \\
6. & $0.02-0.03 i$ & $0.022,0.254,0.604$ & $0.009,0.104,0.289$ & $1.04,1.57,6.03$ \\
\hline S.No. & $\mathrm{Y}_{1}$ & $Y_{\nu}$ & $m_{\nu}(\mathrm{eV})$ & $M_{\nu^{c}}$ \\
\hline 1. & $0.007,0.026,0.421$ & $0.014,0.032,0.533$ & $0.16,0.1597,0.0104,0.0104,1.7 \times 10^{-6}$ & $365.07,0,0$ \\
2. & $0.023,0.094,0.314$ & $0.018,0.213,0.566$ & $0.2056,0.2054,0.05,0.0499,3.8 \times 10^{-6}$ & $365.07,0,0$ \\
3. & $0.031,0.103,0.212$ & $0.015,0.187,0.624$ & $0.3021,0.302,0.0781,0.0781,4.4 \times 10^{-7}$ & $365.07,0,0$ \\
4. & $0.029,0.094,0.259$ & $0.018,0.283,0.42$ & $0.1806,0.1805,0.1254,0.1254,7.8 \times 10^{-7}$ & $365.07,0,0$ \\
5. & $0.009,0.073,0.4$ & $0.008,0.214,0.558$ & $0.1946,0.1945,0.0533,0.0532,7.2 \times 10^{-7}$ & $365.07,0,0$ \\
6. & $0.007,0.148,0.338$ & $0.01,0.159,0.608$ & $0.2837,0.2836,0.0129,0.0128,6.4 \times 10^{-6}$ & $365.07,0,0$ \\
\hline
\end{tabular}

TABLE IV: Yukawa eigenvalues and mixing angles for $N_{g}=3(\operatorname{Rank}[\boldsymbol{\Xi}]=5), f=0.9+0.7 i$. $\lambda=.48+.3 i, \eta=.25, h=1.3, \gamma=.05, \bar{\gamma}=-1.2, \xi=3.645+.363 i . M_{\nu^{c}}$ is independent of $M_{H}$ value chosen. Notice the four neutrino masses in the $10^{1 \div 2} \mathrm{meV}$ range. 


\begin{tabular}{|c|c|c|c|}
\hline $\begin{array}{c}\text { Field } \\
{[\mathrm{SU}(3), \mathrm{SU}(2), \mathrm{Y}]}\end{array}$ & Masses & $\begin{array}{c}\text { Field } \\
{[\mathrm{SU}(3), \mathrm{SU}(2), \mathrm{Y}]}\end{array}$ & Masses \\
\hline$A[1,1,4]$ & $9.93,8.08,7.34,4.06,2.53,0.84$ & $B[6,2,5 / 3]$ & $6.46,6.38,5.8,5.18,4.83,2.88$ \\
\hline$C[8,2,1]$ & $4.62,4.62,4.36,4.36,4 ., 4$. & $C[8,2,1]$ & $2.2,2.2,1.88,1.88,0.35,0.35$ \\
\hline$D[3,2,7 / 3]$ & $7.55,4.64,4.25,3.95,2.71,2$ & $D[3,2,7 / 3]$ & $1.92,1.61,1.39,1.1,1.08,0.53$ \\
\hline$E[3,2,1 / 3]$ & $28.48,28.48,20.62,19.48,18.45$ & $E[3,2,1 / 3]$ & $18.19,14.85,13.38,5.94,4.47,3.32$ \\
\hline$E[3,2,1 / 3]$ & $2.87,2.54,2.28,2.2,1.54,1.36,1.28$ & $E[3,2,1 / 3]$ & $1.16,1.06,0.97,0.8,0.4,0.25,0.24$ \\
\hline$F[1,1,2]$ & $24.66,24.66,21.78,20.8,19.4,18.4$ & $F[1,1,2]$ & $14.65,13.88,4.6,3.65,1.9,1.7,0.14$ \\
\hline$G[1,1,0]$ & $45.55,45.55,39.11,37.34,36.03$ & $G[1,1,0]$ & $35.82,27.46,26.75,15.96,14.2,14.0$ \\
\hline$G[1,1,0]$ & $13.83,11.92,11.41,10.75,8.62$ & $G[1,1,0]$ & $7.15,6.35,6.03,5.27,4.47,3.4,2.15$ \\
\hline$G[1,1,0]$ & $1.77,0.76,0.66,0.11,0.05,0,0,0$ & $I[3,1,10 / 3]$ & $17.23,13.25,11.82,4.61,2.61,1.17$ \\
\hline$J[3,1,4 / 3]$ & $25.09,25.09,23.7,23.02,20.65,19.5$ & $J[3,1,4 / 3]$ & $16.49,15.45,7.68,7.07,5.18,4.66$ \\
\hline$J[3,1,4 / 3]$ & $4.51,3.25,3.07,1.94,0.86,0.68,0.23$ & $K[3,1,-8 / 3]$ & $8.41,5.21,4.54,1.46,1.01,0.44$ \\
\hline$L[6,1,2 / 3]$ & $13.27,9.28,5.3,4.92,3.49,0.91$ & $M[6,1,8 / 3]$ & $12.48,8.46,6.42,4.37,3.29,0.71$ \\
\hline$N[6,1,-4 / 3]$ & $14.23,10.71,7.19,7.07,3.87,0.22$ & $O[1,3,-2]$ & $14.05,9.35,8.34,5.48,3.22,1.05$ \\
\hline$P[3,3,-2 / 3]$ & $9.63,7.14,5.65,2.8,1.6,1.27$ & $Q[8,3,0]$ & $15.14,12.38,8.53,7.75,2.31,0.93$ \\
\hline$R[8,1,0]$ & $27.29,18.82,13.31,10.7,9.3,6.36$ & $R[8,1,0]$ & $6.12,6.07,5.45,3.68,2.58,0.15$ \\
\hline$S[1,3,0]$ & $29.76,19.87,14.44,10.53,7.96,1.74$ & $U[3,3,4 / 3]$ & $24.78,15.76,13.17,6.8,5.83,3.13$ \\
\hline$V[1,2,-3]$ & $18.19,16.8,15.59,13.91,4.48,2.43$ & $W[6,3,2 / 3]$ & $6.72,5.37,4.41,2.72,1.44,0.15$ \\
\hline$X[3,2,-5 / 3]$ & $16.48,16.48,11.8,9.05,8.37,6.22$ & $X[3,2,-5 / 3]$ & $3.69,3.5,2.79,1.91,1.31,0.77,0.49$ \\
\hline$Y[6,2,-1 / 3]$ & $7.51,7 ., 6.1,3.69,2.2,1.52$ & $Z[8,1,2]$ & $27.05,18.02,10.94,9.02,8.06,0.93$ \\
\hline
\end{tabular}

TABLE V: Mass Spectrum of superheavy fields in units of $m / \lambda \sim 10^{16} \mathrm{GeV}$ in six-dimensional $\operatorname{symmetric} \operatorname{tracefull}(\operatorname{Rank}[\boldsymbol{\Xi}]=5)$ scenario with $N_{g}=3$ for the solution described in Section IV.B.1. Only the spectra of $h[1,2, \pm 1], t[3,1, \mp 2 / 3]$ depend on the value of $M_{H}$ chosen. See Table VI for the spectra of $h[1,2, \pm 1], t[3,1, \mp 2 / 3]$ type multiplets for each of the six values of $M_{H}$. 


\begin{tabular}{|c|c|c|}
\hline$M_{H}$ & $h[1,2,1]$ & $t[3,1,-2 / 3]$ \\
\hline \multirow[t]{4}{*}{$2.55+0.13 i$} & $34.81,32.15,27.12,21.08,20.53$ & $39 ., 36.27,35.17,27.86,27.6,24.06,21.67$ \\
\hline & $18.99,12.6,12.06,11.62,10.96,10.38$ & $18.22,13.95,12.81,10.72,8.94,8.28,7.46,6.5$ \\
\hline & $9.51,7.29,6.87,3.57,2.7,2.28,2.06$ & $5.07,4.49,4.09,3.44,3.08,2.23,1.52,1.36$ \\
\hline & $0.74,0.59,0.32,0.13,0.05$ & $0.78,0.49,0.43,0.26,0.21,0.11,0.04$ \\
\hline \multirow[t]{4}{*}{$1.44-0.61 i$} & $34.77,32.11,27.08,21.06,20.5,18.95$ & $38.97,36.25,35.14,27.88,27.49,24.01,21.68$ \\
\hline & $12.5,11.96,11.55,10.81,10.28,9.54$ & $18.09,13.86,12.87,10.71,8.73,8.17,7.54$ \\
\hline & $7.3,6.87,3.5,2.59,2.31,1.97$ & $6.53,5.05,4.49,4.07,3.45,2.92,2.24,1.49$ \\
\hline & $0.5,0.38,0.23,0.11,0.04$ & $1.29,0.61,0.44,0.37,0.23,0.18,0.1,0.05$ \\
\hline \multirow[t]{4}{*}{$1.28+0.75 i$} & $34.77,32.1,27.08,21.05,20.5,18.95$ & $38.97,36.25,35.13,27.88,27.48,24 ., 21.69,18.08$ \\
\hline & $12.49,11.95,11.55,10.81,10.27,9.54$ & $13.86,12.87,10.7,8.72,8.16,7.55,6.53,5.05$ \\
\hline & $7.3,6.86,3.5,2.59,2.32,1.96$ & $4.49,4.07,3.45,2.91,2.24,1.49,1.29$ \\
\hline & $0.49,0.37,0.22,0.11,0.01$ & $0.58,0.44,0.35,0.21,0.17,0.1,0.06$ \\
\hline \multirow[t]{4}{*}{$1.16+0.67 i$} & $34.77,32.1,27.07,21.05,20.49,18.94$ & $38.96,36.24,35.13,27.88,27.47,24 ., 21.69,18.07$ \\
\hline & $12.48,11.94,11.54,10.79,10.26,9.55$ & $13.85,12.88,10.7,8.7,8.15,7.56,6.54,5.05$ \\
\hline & $7.3,6.86,3.49,2.58,2.32,1.95$ & $4.49,4.07,3.46,2.89,2.24,1.49,1.28$ \\
\hline & $0.45,0.34,0.21,0.12,0.01$ & $0.55,0.43,0.33,0.21,0.16,0.1,0.06$ \\
\hline \multirow[t]{4}{*}{$1.06-0.73 i$} & $34.77,32.1,27.07,21.05,20.49,18.94$ & $38.96,36.24,35.13,27.89,27.46,24 ., 21.69,18.07$ \\
\hline & $12.48,11.94,11.54,10.79,10.25,9.55$ & $13.85,12.88,10.7,8.69,8.15,7.56,6.54,5.05$ \\
\hline & $7.31,6.86,3.49,2.58,2.32,1.95$ & $4.49,4.07,3.45,2.89,2.24,1.49,1.28$ \\
\hline & $0.46,0.34,0.22,0.14,0.03$ & $0.54,0.43,0.34,0.21,0.16,0.1,0.06$ \\
\hline \multirow[t]{4}{*}{$0.02-0.03 i$} & $34.75,32.08,27.05,21.04,20.47,18.92$ & $38.95,36.24,35.12,27.91,27.4,23.97,21.69,18$ \\
\hline & $12.44,11.9,11.51,10.74,10.19,9.57$ & $13.82,12.92,10.68,8.58,8.1,7.6,6.56,5.05$ \\
\hline & $7.33,6.86,3.48,2.57,2.33,1.9$ & $4.5,4.06,3.46,2.84,2.24,1.48,1.25$ \\
\hline & $0.36,0.28,0.23,0.21,0.09$ & $0.43,0.31,0.22,0.19,0.13,0.11,0.06$ \\
\hline
\end{tabular}

TABLE VI: Mass Spectrum of superheavy fields $h[1,2, \pm 1], t[3,1, \mp 2 / 3]$ which depend on the value of $M_{H}$ chosen in units of $m / \lambda \sim 10^{16} \mathrm{GeV}$ in six-dimensional symmetric tracefull $(\operatorname{Rank}[\boldsymbol{\Xi}]=5)$ scenario with $N_{g}=3$ for each of the solutions described in Section IV.B.1. 


\begin{tabular}{|c|c|c|c|c|c|}
\hline S.No. & $\mathrm{M}_{\mathrm{H}}$ & $\mathrm{Y}_{\mathrm{u}}$ & $\mathrm{Y}_{\mathrm{d}}$ & $\begin{array}{c}\left\{\theta_{13}, \theta_{12}, \theta_{23}\right\}^{\mathrm{Q}} \\
(\text { deg. })\end{array}$ & $m_{\nu} / 10^{-4}(\mathrm{meV})$ \\
\hline 1. & $-4.323+1.47 i$ & $.0007, .0021, .0215$ & $.001, .0019, .0219$ & $9.0,15.9,15.6$ & $0.039,0.187,40.543$ \\
2. & $.465+3.382 i$ & $.0018, .0148, .0182$ & $.0020, .0197, .0222$ & $11.3,1.5,4.9$ & $0.079,0.807,4.09$ \\
3. & $.76-2.193 i$ & $.0029, .0113, .0137$ & $.0054, .0233, .0385$ & $1.5,6.2,7.4$ & $0.12,0.32,6.946$ \\
4. & $-0.002+0.968 i$ & $.0105, .040, .077$ & $.0035, .0174, .0408$ & $5.2,3.7,2.8$ & $1.33,4.503,23.439$ \\
5. & $-.508-.209 i$ & $.0077, .053, .1126$ & $.0019, .0159, .0381$ & $1.1,12.1,1.4$ & $0.926,17.046,34.448$ \\
6. & $-.092-.032 i$ & $.0041, .0467, .0558$ & $.0035, .0413, .0522$ & $8.7,5.5,2.6$ & $1.03,4.96,9.806$ \\
\hline S.No. & $\mathrm{Y}_{1}$ & $Y_{\nu}$ & $\left\{\theta_{13}, \theta_{12}, \theta_{23}\right\}^{\mathrm{L}}$ & $\tilde{M}_{\nu^{c}}$ & $\Delta m_{\nu}^{2} / 10^{-13}(\mathrm{eV})$ \\
\hline 1. & $.0013, .0041, .0517$ & $.0023, .0064, .0468$ & $3.6,20.3,23.3$ & $5.9,5.3,1.5$ & $.0033,164.372$ \\
2. & $.0034, .0148, .0205$ & $.0032, .0126, .0162$ & $27.5,14.5,47.0$ & $5.9,5.3,1.5$ & $0.0645,1.6074$ \\
3. & $.0053, .0121, .0458$ & $.0033, .0102, .020$ & $13.6,11.1,41.1$ & $5.9,5.3,1.5$ & $0.0088,4.8143$ \\
4. & $.0048, .0174, .0473$ & $.0092, .0181, .0915$ & $23.9,14.5,17.7$ & $5.9,5.3,1.5$ & $1.851,52.9128$ \\
5. & $.0042, .0224, .0382$ & $.0061, .0584, .0835$ & $23.7,26.1,49.4$ & $5.9,5.3,1.5$ & $28.9722,89.6078$ \\
6. & $.0043, .0497, .0621$ & $.0049, .0355, .0518$ & $14.1,37.6,46.3$ & $5.9,5.3,1.5$ & $2.3544,7.1562$ \\
\hline
\end{tabular}

TABLE VII: Yukawa eigenvalues and mixing angles for $N_{g}=3(\operatorname{Rank}[\boldsymbol{\Xi}]=4), f=-0.11+.02 i$. $\tilde{M}_{\nu^{c}} \equiv \lambda M_{\nu^{c}} / m . m / \lambda$ is taken to be $10^{16} \mathrm{GeV}$ to estimate $\Delta m_{\nu}^{2} . \quad \lambda=0.48-.05 i, \eta=-.18$, $h=.26, \gamma=0.12, \bar{\gamma}=-1.44$ and $\xi=1.7278-0.1734 i$ 


\begin{tabular}{|c|c|c|c|c|c|}
\hline S.No. & $\mathrm{M}_{\mathrm{H}}$ & $\mathrm{Y}_{\mathrm{u}}$ & $\mathrm{Y}_{\mathrm{d}}$ & $\begin{array}{c}\left\{\theta_{13}, \theta_{12}, \theta_{23}\right\}^{\mathrm{Q}} \\
(\text { deg. })\end{array}$ & $m_{\nu}(\mathrm{meV})$ \\
\hline 1. & $-4.323+1.47 i$ & $0.001,0.003,0.026$ & $0.001,0.003,0.027$ & $0.002,0.008,0.015$ & $0.0006,0.0047,1.2427$ \\
2. & $.465+3.382 i$ & $0.002,0.013,0.015$ & $0.002,0.015,0.017$ & $0.014,0.002,0.003$ & $0.0056,0.0284,0.3286$ \\
3. & $.76-2.193 i$ & $0.003,0.01,0.014$ & $0.005,0.019,0.028$ & $0.002,0.008,0.013$ & $0.0096,0.0256,0.3686$ \\
4. & $-0.002+0.968 i$ & $0.007,0.033,0.079$ & $0.004,0.017,0.042$ & $0.005,0.004,0.002$ & $0.0652,0.8982,3.3757$ \\
5. & $-.508-.209 i$ & $0.006,0.047,0.105$ & $0.002,0.017,0.038$ & $0.001,0.013,0.002$ & $0.0701,2.2717,3.2128$ \\
6. & $-.092-.032 i$ & $0.003,0.044,0.054$ & $0.003,0.043,0.053$ & $0.009,0.006,0.004$ & $0.025,0.7852,1.4609$ \\
\hline S.No. & $\mathrm{Y}_{1}$ & $Y_{\nu}$ & $\left\{\theta_{13}, \theta_{12}, \theta_{23}\right\}^{\mathrm{L}}$ & $\tilde{M}_{\nu^{c}}$ & $\Delta m_{\nu}^{2} / 10^{-5}(\mathrm{eV})$ \\
\hline 1. & $0.001,0.003,0.027$ & $0.001,0.003,0.026$ & $0.27,9.82,2.13$ & $0.006,0.005,0.001$ & $2.16 \times 10^{-6}, 0.15$ \\
\hline. & $0.002,0.015,0.017$ & $0.002,0.013,0.015$ & $2.41,22.56,37.83$ & $0.006,0.005,0.001$ & $7.74 \times 10^{-5}, 0.011$ \\
3. & $0.005,0.019,0.028$ & $0.003,0.01,0.014$ & $2.97,28.08,18.45$ & $0.006,0.005,0.001$ & $5.64 \times 10^{-5}, 0.013$ \\
4. & $0.004,0.017,0.042$ & $0.007,0.033,0.079$ & $5.81,7.17,25.52$ & $0.006,0.005,0.001$ & $0.080,1.06$ \\
5. & $0.002,0.017,0.038$ & $0.006,0.047,0.105$ & $2.7,6.33,54.28$ & $0.006,0.005,0.001$ & $0.516,0.516$ \\
6. & $0.003,0.043,0.053$ & $0.003,0.044,0.054$ & $3.38,7.5,58.18$ & $0.006,0.005,0.001$ & $0.062,0.152$ \\
\hline
\end{tabular}

TABLE VIII: (Truncated) Yukawa eigenvalues and mixing angles for $N_{g}=3$ and the same parameter values as in Table VII but with much smaller $f=-0.00011+.00002 i . \tilde{M}_{\nu^{c}} \equiv \lambda M_{\nu^{c}} / m . m / \lambda$ is taken to be $10^{16} \mathrm{GeV}$ to estimate $\Delta m_{\nu}^{2}$. 


\begin{tabular}{|c|c|c|c|}
\hline $\begin{array}{c}\text { Field } \\
{[\mathrm{SU}(3), \mathrm{SU}(2), \mathrm{Y}]}\end{array}$ & Masses & $\begin{array}{c}\text { Field } \\
{[\mathrm{SU}(3), \mathrm{SU}(2), \mathrm{Y}]}\end{array}$ & Masses \\
\hline$A[1,1,4]$ & $1.53,1.46,1.28,1.26,0.38,0.07$ & $B[6,2,5 / 3]$ & $2.89,2.44,2.41,1.9,1.22,1$ \\
\hline$C[8,2,1]$ & $1.2,1.2,0.91,0.91,0.89,0.89$ & $C[8,2,1]$ & $0.63,0.63,0.6,0.6,0.59,0.59$ \\
\hline$D[3,2,7 / 3]$ & $1.27,0.9,0.86,0.72,0.67,0.63$ & $D[3,2,7 / 3]$ & $0.61,0.51,0.41,0.35,0.19,0.13$ \\
\hline$E[3,2,1 / 3]$ & $6.26,6.26,4.38,3.93,3.54,2.99$ & $E[3,2,1 / 3]$ & $2.94,2.51,1.44,1.12,1.09,1.02$ \\
\hline$E[3,2,1 / 3]$ & $0.97,0.92,0.77,0.73,0.7,0.68,0.6$ & $E[3,2,1 / 3]$ & $0.42,0.32,0.24,0.22, \quad 0.19,0.12$ \\
\hline$F[1,1,2]$ & $6.01,6.01,5.12,3.97,3.44,3.18,2.95$ & $F[1,1,2]$ & $2.77,1.42,1.04,0.46, \quad 0.19,0.12$ \\
\hline$G[1,1,0]$ & $10.19,10.19,8.23,7.9,6.58,6.27$ & $G[1,1,0]$ & $5.52,5 ., 3.99,3.75,2.88,2.79$ \\
\hline$G[1,1,0]$ & $2.53,2.22,2.15,2.03,1.79,1.71$ & $G[1,1,0]$ & $1.67,1.33,0.97,0.95,0.88,0.79$ \\
\hline$G[1,1,0]$ & $0.62,0.42,0.06,0,0,0,0$ & $I[3,1,10 / 3]$ & $2.89,2.74,2.42,2.37,1.46,0.3$ \\
\hline$J[3,1,4 / 3]$ & $6.4,6.4,4.68,3.93,3.45,3.09$ & $J[3,1,4 / 3]$ & $2.97,2.64,1.68,1.27,1.19,1.14$ \\
\hline$J[3,1,4 / 3]$ & $0.96,0.9,0.54,0.37,0.16,0.11,0.03$ & $K[3,1,-8 / 3]$ & $1.01,0.77,0.76,0.63,0.56,0.47$ \\
\hline$L[6,1,2 / 3]$ & $1.53,1.08,1.04,0.63,0.61,0.6$ & $M[6,1,8 / 3]$ & $1.58,1.33,1.27,1.05,0.78,0.39$ \\
\hline$N[6,1,-4 / 3]$ & $1.63,1.31,0.98,0.93,0.7,0.49$ & $O[1,3,-2]$ & $1.36,1.22,0.81,0.7,0.49,0.22$ \\
\hline$P[3,3,-2 / 3]$ & $0.8,0.7,0.62,0.36,0.19,0.18$ & $Q[8,3,0]$ & $2.89,2.69,2.09,1.59,1.26,1.24$ \\
\hline$R[8,1,0]$ & $3.54,2.89,2.66,2.52,2.43,1.9$ & $R[8,1,0]$ & $1.78,1.53,1.32,0.89,0.88,0.77$ \\
\hline$S[1,3,0]$ & $2.92,1.89,1.51,0.89,0.84,0.51$ & $U[3,3,4 / 3]$ & $1.52,1.48,1.17,0.96,0.28,0.26$ \\
\hline$V[1,2,-3]$ & $3.77,2.59,1.96,0.96,0.86,0.77$ & $W[6,3,2 / 3]$ & $1.27,1.1,1 ., 0.83,0.73,0.7$ \\
\hline$X[3,2,-5 / 3]$ & $2.89,2.33,2.33,2.08,1.8,1.75$ & $X[3,2,-5 / 3]$ & $1.37,1.06,0.96,0.92,0.88, \quad 0.87,0.34$ \\
\hline$Y[6,2,-1 / 3]$ & $2.51,1.74,1.68,0.97,0.91,0.89$ & $Z[8,1,2]$ & $3.39,2.18,2.12,1.16,0.98,0.96$ \\
\hline
\end{tabular}

TABLE IX: Mass Spectrum of superheavy fields in units of $m / \lambda \sim 10^{16} \mathrm{GeV}$ in six-dimensional symmetric tracefull scenario $(\operatorname{Rank}[\boldsymbol{\Xi}]=4)$ for $N_{g}=3$ for the solution described in Section IV.B.2. Only the spectra of $h[1,2, \pm 1], t[3,1, \mp 2 / 3]$ depend on the value of $M_{H}$ chosen. See Table $\mathrm{X}$ for these spectra. 


\begin{tabular}{|c|c|c|}
\hline$M_{H}$ & $h[1,2,1]$ & $t[3,1,-2 / 3]$ \\
\hline \multirow[t]{4}{*}{$-4.323+1.47 i$} & $10.09,9.86,8.22,7.93,7.31,6.46$ & $9.96,9.82,7.84,7.49,7.12,6.57,5.94$ \\
\hline & $5.85,3.74,3.49,3.26,2.39,2.04$ & $3.43,3.29,3.05,2.22,1.84,1.72,1.49,1.32,1.16$ \\
\hline & $1.76,1.41,0.89,0.75,0.63,0.61$ & $1.05,1 ., 0.99,0.79,0.74,0.65,0.63,0.61$ \\
\hline & $0.48,0.33,0.27,0.1,0.03$ & $0.52,0.4,0.32,0.28,0.2,0.12$ \\
\hline \multirow[t]{4}{*}{$.465+3.382 i$} & $9.71,9.48,7.76,7.42,6.71,6.27,5.16$ & $9.63,9.39,7.35,7.03,6.59,6.28,5.11$ \\
\hline & $3.58,3.17,2.64,2.4,1.96,1.72$ & $3.18,2.91,2.7,2.1,1.83,1.68,1.46,1.36$ \\
\hline & $1.55,0.92,0.66,0.63,0.61$ & $1.21,1.11,1.04,1.02,0.8,0.74,0.66,0.63$ \\
\hline & $0.52,0.32,0.26,0.13,0.03$ & $0.61,0.49,0.37,0.25,0.22,0.17,0.13$ \\
\hline \multirow[t]{4}{*}{$.76-2.193 i$} & $9.52,9.22,7.52,7.1,6.35,5.94$ & $9.46,9.09,7.14,6.74,6.23,5.87,4.46,3.01$ \\
\hline & $4.61,3.29,2.87,2.52,2.09,1.92$ & $2.51,2.14,2.09,1.91,1.72,1.43,1.28,1.2$ \\
\hline & $1.76,1.17,0.97,0.72,0.66,0.62$ & $1.12,1.07,1.03,0.79,0.77,0.63,0.61$ \\
\hline & $0.52,0.31,0.22,0.17,0.04$ & $0.59,0.47,0.35,0.27,0.18,0.14,0.1$ \\
\hline \multirow[t]{4}{*}{$-0.002+0.968 i$} & $9.4,9.01,7.38,6.9,6.16,5.7$ & $9.34,8.87,7.04,6.54,5.98,5.61,3.84,2.83$ \\
\hline & $4.19,3.18,2.61,2.36,2.11,1.38$ & $2.25,1.99,1.83,1.69,1.64,1.38,1.32,1.29$ \\
\hline & $1.15,1.07,1.01,0.73,0.66,0.63$ & $1.17,1.06,0.95,0.82,0.79,0.63,0.61$ \\
\hline & $0.36,0.2,0.13,0.06,0.02$ & $0.57,0.38,0.2,0.13,0.1,0.08,0.03$ \\
\hline \multirow[t]{4}{*}{$-.508-.209 i$} & $9.39,8.99,7.37,6.86,6.15,5.64$ & $9.33,8.84,7.03,6.5,5.96,5.54,3.76,2.84$ \\
\hline & $4.12,3.16,2.45,2.41,2.13,1.35$ & $2.1,1.99,1.79,1.7,1.62,1.46,1.35,1.27$ \\
\hline & $1.24,0.95,0.82,0.71,0.67,0.63$ & $1.14,0.98,0.91,0.82,0.81,0.63,0.59$ \\
\hline & $0.39,0.17,0.11,0.05,0.02$ & $0.55,0.28,0.14,0.12,0.08,0.06,0.03$ \\
\hline \multirow[t]{4}{*}{$-.092-.032 i$} & $9.37,8.98,7.35,6.84,6.13,5.61$ & $9.32,8.82,7.03,6.48,5.95,5.52,3.71,2.83$ \\
\hline & $4.1,3.17,2.45,2.37,2.17,1.32$ & $2.07,1.99,1.82,1.7,1.62,1.45,1.33$ \\
\hline & $1.2,0.88,0.85,0.72,0.68,0.63$ & $1.27,1.14,1 ., 0.89,0.83,0.81,0.63,0.59$ \\
\hline & $0.37,0.12,0.08,0.05,0.02$ & $0.55,0.31,0.1,0.07,0.06,0.04,0.02$ \\
\hline
\end{tabular}

TABLE X: Mass Spectrum of superheavy fields $h[1,2, \pm 1], t[3,1, \mp 2 / 3]$ which depend on the value of $M_{H}$ chosen in units of $m / \lambda \sim 10^{16} \mathrm{GeV}$ in six-dimensional symmetric tracefull $(\operatorname{Rank}[\boldsymbol{\Xi}]=4)$ scenario with $N_{g}=3$ for each of the solutions described in Section IV.B.2. 


\begin{tabular}{|c|c|c|c|c|c|}
\hline S.No. & $\mathrm{M}_{\mathrm{H}}$ & $\mathrm{Y}_{\mathrm{u}}$ & $\mathrm{Y}_{\mathrm{d}}$ & $\begin{array}{c}\left\{\theta_{13}, \theta_{12}, \theta_{23}\right\}^{\mathrm{Q}} \\
(\text { deg. })\end{array}$ & $m_{\nu}(m e V)$ \\
\hline 1. & $135.29+11.98 i$ & $.054, .068, .1718$ & $.0001, .0012, .0019$ & $8.23,34.35,32.04$ & $(1.29,1.62,4.1) \times 10^{-2}$ \\
\hline 2. & $25.4+1.72 i$ & $0.0, .0588, .0938$ & $0.0, .0021, .0034$ & $(.1,1.7,1.2) \times 10^{-6}$ & $6.5 \times 10^{-11}, .012, .028$ \\
\hline 3. & $24.6+1.16 i$ & $0.0, .0614, .1325$ & $0.0, .0016, .0036$ & $8.12 \times 10^{-8}, 0,0$ & $2.0 \times 10^{-10}, .017, .045$ \\
\hline 4. & $18.41+1.4 i$ & $.001, .0555, .125$ & $.0035, .011, .0264$ & $3.48,6.01,8.67$ & $1.5 \times 10^{-6}, .010, .011$ \\
\hline 5. & $18.32+1.23 i$ & $0.0, .0584, .1322$ & $0.0, .0112, .0255$ & $4.86 \times 10^{-9}, 0,0$ & $1.1 \times 10^{-10}, .012, .013$ \\
\hline S.No. & $\mathrm{Y}_{1}$ & $Y_{\nu}$ & $\begin{array}{c}\left\{\theta_{13}, \theta_{12}, \theta_{23}\right\}^{\mathrm{L}} \\
(\text { deg. })\end{array}$ & $\tilde{M}_{\nu^{c}}$ & $\Delta m_{\nu}^{2}\left(e V^{2}\right)$ \\
\hline 1. & $.0029, .0066, .013$ & $.174, .2183, .551$ & $5.92,32.15,9.6$ & $22.39,8.89,7.05$ & $(.096,1.42) \times 10^{-9}$ \\
\hline 2. & $0.0, .0177, .0283$ & $0.0, .2272, .3629$ & $(.11,8.5) \times 10^{-7}, 28.7$ & $22.39,8.89,7.05$ & $(1.49,6.18) \times 10^{-10}$ \\
\hline 3. & $0.0, .0068, .0147$ & $0.0, .236, .5094$ & $(.03,1.2) \times 10^{-6}, 19.4$ & $22.39,8.89,7.05$ & $(2.75,17.9) \times 10^{-10}$ \\
\hline 4. & $.0098, .0136, .038$ & $.0028, .122, .275$ & $14.33,20.53,35.13$ & $22.39,8.89,7.05$ & $(1.08, .23) \times 10^{-10}$ \\
\hline 5 . & $0.0, .015, .034$ & $0.0, .1284, .291$ & $5.7 \times 10^{-9}, 0,42.03$ & $22.39,8.89,7.05$ & $(1.33, .29) \times 10^{-10}$ \\
\hline
\end{tabular}

TABLE XI: Yukawa eigenvalues and mixing angles for $N_{g}=3(\operatorname{Rank}[\boldsymbol{\Xi}]=4), f=0.23+.04 i$. $\tilde{M}_{\nu^{c}} \equiv \lambda M_{\nu^{c}} / m . m / \lambda$ is taken to be $10^{16} \mathrm{GeV}$ to estimate $\Delta m_{\nu}^{2} \cdot \lambda=0.18-.03 i, \eta=.034, \gamma=$ $-0.53, \bar{\gamma}=-2.60, h=.14$ and $\xi=7.677+0.15772 i . M_{\nu^{c}}$ is independent of $M_{H}$ value chosen. 


\begin{tabular}{|c|c|c|c|}
\hline $\begin{array}{c}\text { Field } \\
{[\mathrm{SU}(3), \mathrm{SU}(2), \mathrm{Y}]}\end{array}$ & Masses & $\begin{array}{c}\text { Field } \\
{[\mathrm{SU}(3), \mathrm{SU}(2), \mathrm{Y}]}\end{array}$ & Masses \\
\hline$A[1,1,4]$ & $0.602,0.596,0.515,0.461,0.446$ & $B[6,2,5 / 3]$ & $1.099,0.861,0.826,0.248,0.244$ \\
\hline$C[8,2,1]$ & $0.563,0.563,0.552,0.552,0.532$ & $C[8,2,1]$ & $0.532,0.483,0.483,0.482,0.482$ \\
\hline$D[3,2,7 / 3]$ & $0.584,0.571,0.563,0.561,0.531$ & $D[3,2,7 / 3]$ & $0.513,0.493,0.482,0.465,0.461$ \\
\hline$E[3,2,1 / 3]$ & $8.843,8.843,1.48,1.47,0.92$ & $E[3,2,1 / 3]$ & $0.858,0.847,0.658,0.658,0.482$ \\
\hline$E[3,2,1 / 3]$ & $0.467,0.462,0.452,0.426,0.423$ & $E[3,2,1 / 3]$ & $0.388,0.351,0.33,0.31,0.061,0.022$ \\
\hline$F[1,1,2]$ & $8.262,8.262,1.603,1.591,0.997$ & $F[1,1,2]$ & $0.901,0.882,0.49,0.462,0.165,0.075$ \\
\hline$G[1,1,0]$ & $15.3,15.3,2.62,2.601,1.866$ & $G[1,1,0]$ & $1.317,1.315,1.111,1.11,0.979$ \\
\hline$G[1,1,0]$ & $0.92,0.772,0.744,0.642,0.626$ & $G[1,1,0]$ & $0.498,0.384,0.327,0.327,0.245$ \\
\hline$G[1,1,0]$ & $0.136,0.065,0.031,0,0,0$ & $I[3,1,10 / 3]$ & $0.974,0.898,0.462,0.149,0.06$ \\
\hline$J[3,1,4 / 3]$ & $9.188,9.188,1.438,1.428,0.908$ & $J[3,1,4 / 3]$ & $0.83,0.821,0.573,0.525,0.494,0.469$ \\
\hline$J[3,1,4 / 3]$ & $0.432,0.21,0.19,0.07,0.025$ & $K[3,1,-8 / 3]$ & $0.565,0.555,0.53,0.483,0.48$ \\
\hline$L[6,1,2 / 3]$ & $0.596,0.574,0.542,0.452,0.45$ & $M[6,1,8 / 3]$ & $0.692,0.635,0.582,0.359,0.356$ \\
\hline$N[6,1,-4 / 3]$ & $0.549,0.548,0.516,0.503,0.496$ & $O[1,3,-2]$ & $0.772,0.771,0.419,0.396,0.282$ \\
\hline$P[3,3,-2 / 3]$ & $0.596,0.593,0.504,0.471,0.45$ & $Q[8,3,0]$ & $0.967,0.846,0.595,0.14,0.112$ \\
\hline$R[8,1,0]$ & $1.18,0.959,0.856,0.441,0.406$ & $R[8,1,0]$ & $0.394,0.319,0.31,0.274,0.272$ \\
\hline$S[1,3,0]$ & $1.279,1.269,0.537,0.098,0.024$ & $U[3,3,4 / 3]$ & $0.776,0.762,0.3,0.138,0.051$ \\
\hline$V[1,2,-3]$ & $1.065,1.05,0.422,0.167,0.076$ & $W[6,3,2 / 3]$ & $0.63,0.602,0.549,0.421,0.416$ \\
\hline$X[3,2,-5 / 3]$ & $2.084,2.084,0.857,0.791,0.772$ & $X[3,2,-5 / 3]$ & $0.699,0.577,0.238,0.16,0.07,0.053$ \\
\hline$Y[6,2,-1 / 3]$ & $0.587,0.533,0.426,0.167,0.158$ & $Z[8,1,2]$ & $0.768,0.669,0.501,0.063,0.045$ \\
\hline
\end{tabular}

TABLE XII: Mass Spectrum of superheavy fields in units of $m / \lambda \sim 10^{16} \mathrm{GeV}$ in the fivedimensional symmetric traceless case $\left(N_{g}=3, \operatorname{Rank}[\boldsymbol{\Xi}]=4\right)$, for the solution described in Section IV.C. Only the spectra of $h[1,2, \pm 1], t[3,1, \mp 2 / 3]$ depend on the value of $M_{H}$ chosen. See TableXII] for these spectra. 


\begin{tabular}{|c|c|c|}
\hline$M_{H}$ & $h[1,2,1]$ & $t[3,1,-2 / 3]$ \\
\hline \multirow[t]{4}{*}{$135.29+11.98 i$} & $136.697,136.68,136.528,135.959$ & $136.517,136.501,136.392,135.942,135.923,2.293$ \\
\hline & $135.936,2.939,2.925,1.921,0.78,0.77$ & $2.282,1.407,0.697,0.687,0.652,0.609,0.593$ \\
\hline & $0.655,0.62,0.557,0.55,0.505,0.481$ & $0.573,0.559,0.527,0.522,0.52,0.483$ \\
\hline & $0.466,0.266,0.217$ & $0.477,0.391,0.387,0.169,0.139,0.072$ \\
\hline \multirow[t]{4}{*}{$25.4+1.72 i$} & $29.744,29.664,28.968,26.179,26.063$ & $28.908,28.83,28.323,26.094,25.996,2.715$ \\
\hline & $3.287,3.252,1.821,1.52$ & $2.688,1.506,1.195,0.86,0.809,0.679$ \\
\hline & $0.992,0.927,0.658,0.605,0.579$ & $0.633,0.599,0.572,0.556,0.534,0.526,0.398$ \\
\hline & $0.517,0.284,0.115,0.103,0.008$ & $0.392,0.258,0.177,0.161,0.026,0.007$ \\
\hline \multirow[t]{4}{*}{$24.6+1.16 i$} & $29.034,28.952,28.237,25.37,25.25$ & $28.176,28.096,27.575,25.282,25.181,2.732$ \\
\hline & $3.299,3.263,1.828,1.55$ & $2.703,1.533,1.202,0.868,0.816,0.682$ \\
\hline & $1.003,0.937,0.659,0.606,0.585$ & $0.635,0.599,0.572,0.556,0.534,0.526,0.398$ \\
\hline & $0.518,0.285,0.104,0.092,0.008$ & $0.392,0.259,0.17,0.153,0.031,0.012$ \\
\hline \multirow[t]{4}{*}{$18.41+1.4 i$} & $24.01,23.909,23.03,19.435,19.281$ & $22.965,22.865,22.217,19.321,19.19,2.89$ \\
\hline & $3.413,3.353,2.055,1.669$ & $2.841,1.831,1.228,0.942,0.885,0.734,0.644$ \\
\hline & $1.115,1.043,0.697,0.633,0.602$ & $0.604,0.577,0.562,0.537,0.528,0.398$ \\
\hline & $0.528,0.291,0.076,0.061,0.004$ & $0.392,0.276,0.109,0.095,0.069,0.061$ \\
\hline \multirow[t]{4}{*}{$18.32+1.23 i$} & $23.926,23.825,22.943,19.332,19.177$ & $22.878,22.777,22.127,19.218,19.086$ \\
\hline & $3.416,3.355,2.062,1.669$ & $2.893,2.844,1.838,1.228,0.943,0.887,0.735$ \\
\hline & $1.118,1.046,0.699,0.633,0.602$ & $0.645,0.604,0.577,0.562,0.537,0.528,0.398$ \\
\hline & $0.528,0.291,0.078,0.062,0.004$ & $0.392,0.276,0.108,0.095,0.069,0.061$ \\
\hline
\end{tabular}

TABLE XIII: Mass Spectrum of superheavy fields $h[1,2, \pm 1], t[3,1, \mp 2 / 3]$ which depend on the value of $M_{H}$ chosen in units of $m / \lambda \sim 10^{16} \mathrm{GeV}$ in five-dimensional symmetric traceless $(\operatorname{Rank}[\boldsymbol{\Xi}]=$ 4) scenario with $N_{g}=3$ for each of the solutions described in Section IV.C. 


\section{Acknowledgements}

C.S.A. is grateful to Borut Bajc for numerous valuable discussions. C.K.K. thanks the University Grants Commission of the Government of India for financial support through an UGC-Senior research fellowship.

[1] C.S. Aulakh, B. Bajc, A. Melfo, A. Rasin and G. Senjanovic, Nucl. Phys. B 597, 89 (2001) arXiv:hep-ph/0004031.

[2] C.S. Aulakh and R.N. Mohapatra, CCNY-HEP-82-4 April 1982, CCNY-HEP-82-4-REV, Jun 1982 , Phys. Rev. D28, 217 (1983).

[3] T.E. Clark, T.K. Kuo, and N. Nakagawa, Phys. lett. 115B, 26(1982).

[4] C.S. Aulakh, B. Bajc, A. Melfo, G. Senjanovic and F. Vissani, Phys. Lett. B 588, 196 (2004) arXiv:hep-ph/0306242.

[5] C.S. Aulakh, From germ to bloom, arXiv:hep-ph/0506291; C.S. Aulakh and S.K. Garg, Nucl. Phys. B 757, 47 (2006) arXiv:hep-ph/0512224.

[6] C.S. Aulakh and S.K. Garg, Nucl. Phys.B857 (2012)101, arXiv:0807.0917v3.

[7] C.S. Aulakh, arXiv:hep-ph/1107.2963 ; C.S. Aulakh, I. Garg and C.K. Khosa, Nucl. Phys. B 882, 397(2014), arXiv:1311.6100 [hep-ph].

[8] N. Cabibbo and L. Maiani, Evolution of particle physics, pages 50-80 (1970). For a useful recent pedagogical review see R.A. de Pablo, arXiv:1307.1904v1[hep-ph].

[9] Y. Koide, Phys. Rev. D 78, 093006 (2008) [arXiv:0809.2449 [hep-ph]]; Phys. Rev. D 79, 033009 (2009) [arXiv:0811.3470 [hep-ph]]; Phys. Lett. B 665, 227 (2008).

[10] C.S. Aulakh and A. Girdhar, Nucl. Phys. B 711, 275 (2005).

[11] C.S. Aulakh and A. Girdhar, Int. J. Mod. Phys. A 20, 865 (2005)

[12] B. Bajc, A. Melfo, G. Senjanovic and F. Vissani, Phys. Rev. D 70, 035007 (2004) arXiv:hep-ph/0402122;

[13] C.S. Aulakh, "Bajc-Melfo Vacua enable YUMGUTs", arXiv:1402.3979

[14] S. Ray, Phys. Lett. B 642 (2006) 137 arXiv:hep-th/0607172.

[15] B. Bajc and A. Melfo, JHEP 0804, 062 (2008) [arXiv:0801.4349 [hep-ph]].

[16] E. Witten, Phys. Lett. B 105 (1981) 267. 
[17] B.A. Ovrut and S. Raby, Phys. Lett. B 125, 270 (1983).

[18] C.S. Aulakh, "Local Supersymmetry And Hi-lo Scale Induction," CCNY-HEP-83/2; C.S. Aulakh, PhD Thesis, City University of New York, 1983, UMI-84-01477.

[19] R. Bernabei, P. Belli, S. d'Angelo, A. Di Marco, F. Montecchia, F. Cappella, A. d'Angelo and A. Incicchitti et al., Int. J. Mod. Phys. A 28, 1330022 (2013) arXiv:1306.1411 [astro-ph.GA]].

[20] G.D. Coughlan, W. Fischler, E.W. Kolb, S. Raby and G. G. Ross, Phys. Lett. B 131 (1983) 59; B. de Carlos, J. A. Casas, F. Quevedo and E. Roulet, Phys. Lett. B 318 (1993) 447 hep-ph/9308325.

[21] C.S. Aulakh, hep-ph/0602132.

[22] S. Bertolini, T. Schwetz and M. Malinsky, Phys. Rev. D 73 (2006) 115012 hep-ph/0605006.

[23] B. Bajc, A. Melfo, G. Senjanovic and F. Vissani, Phys. Lett. B 634, 272 (2006) hep-ph/0511352.

[24] T. Fukuyama, A. Ilakovac, T. Kikuchi, S. Meljanac and N. Okada, Eur. Phys. J. C 42, 191 (2005) arXiv:hep-ph/0401213v1.,v2. 